\title{
PERSISTENCE OF PERIODIC SOLUTIONS FOR HIGHER ORDER PERTURBED DIFFERENTIAL SYSTEMS VIA LYAPUNOV-SCHMIDT REDUCTION
}

\author{
MURILO R. CÂNDIDO ${ }^{2}$, JAUME LLIBRE $^{2}$, AND DOUGLAS D. NOVAES $^{1}$
}

ABSTRACT. In this work we first provide sufficient conditions to assure the persistence of some zeros of functions having the form

$$
g(z, \varepsilon)=g_{0}(z)+\sum_{i=1}^{k} \varepsilon^{i} g_{i}(z)+\mathcal{O}\left(\varepsilon^{k+1}\right),
$$

for $|\varepsilon| \neq 0$ sufficiently small. Here $g_{i}: \mathcal{D} \rightarrow \mathbb{R}^{n}$, for $i=0,1, \ldots, k$, are smooth functions being $\mathcal{D} \subset \mathbb{R}^{n}$ an open bounded set. Then we use this result to compute the bifurcation functions which controls the periodic solutions of the following $T$-periodic smooth differential system

$$
x^{\prime}=F_{0}(t, x)+\sum_{i=1}^{k} \varepsilon^{i} F_{i}(t, x)+\mathcal{O}\left(\varepsilon^{k+1}\right), \quad(t, z) \in \mathbb{S}^{1} \times \mathcal{D} .
$$

It is assumed that the unperturbed differential system has a sub-manifold of periodic solutions $\mathcal{Z}, \operatorname{dim}(\mathcal{Z}) \leq n$. We also study the case when the bifurcation functions have a continuum of zeros. Finally we provide the explicit expressions of the bifurcation functions up to order 5 .

\section{INTRODUCTION}

This work contains two main results. The first one (see Theorem A) provides sufficient conditions to assure the persistence of some zeros of smooth functions $g: \mathbb{R}^{n} \times \mathbb{R} \rightarrow \mathbb{R}^{n}$ having the form

$$
g(z, \varepsilon)=g_{0}(z)+\sum_{i=1}^{k} \varepsilon^{i} g_{i}(z)+\mathcal{O}\left(\varepsilon^{k+1}\right) .
$$

The second one (see Theorem B) provides sufficient conditions to assure the existence of periodic solutions of the following differential system

$$
x^{\prime}=F(t, z, \varepsilon)=F_{0}(t, x)+\sum_{i=1}^{k} \varepsilon^{i} F_{i}(t, x)+\mathcal{O}\left(\varepsilon^{k+1}\right), \quad(t, z) \in \mathbb{S}^{1} \times \mathcal{D} .
$$

2010 Mathematics Subject Classification. 34C29, 34C25, 37G15.

Key words and phrases. Lyapunov-Schmidt reduction, bifurcation theory, periodic solution, limit cycle, nonlinear differential system. 
Here $\mathbb{S}^{1}=\mathbb{R} / T$, for some $T>0$, and the assumption $t \in \mathbb{S}^{1}$ means that the system is $T$-periodic in the variable $t$. As usual $\delta_{1}(\varepsilon)=\mathcal{O}\left(\delta_{2}(\varepsilon)\right)$ means that there exists a constant $c_{0}>0$, which does not depends on $\varepsilon$, such that $\left|\delta_{1}(\varepsilon)\right| \leq c_{0}\left|\delta_{2}(\varepsilon)\right|$ for $\varepsilon$ sufficiently small (see [16]).

It is assumed that either $g(z, 0)$ vanishes in a submanifold of $\mathcal{Z} \subset \mathcal{D}$, or that the unperturbed differential system $x^{\prime}=F_{0}(t, x)$ has a submanifold $\mathcal{Z} \subset \mathcal{D}$ of $T$-periodic solutions. In both cases $\operatorname{dim}(\mathcal{Z}) \leq n$. The second problem can be often reduced to the first problem, standing as its main motivation.

Regarding the first problem, assume that for some $z^{*} \in \mathcal{Z}, g\left(z^{*}, 0\right)=0$. We shall study the persistence of this zero for the function $(1), g(x, \varepsilon)$, assuming that $|\varepsilon| \neq 0$ is sufficiently small. By persistence we mean the existence of continuous branches $\chi(\varepsilon)$ of simple zeros of $g(x, \varepsilon)$ (that is $g(\chi(\varepsilon), \varepsilon)=0$ ) such that $\chi(0)=z^{*}$. It is well known that if the $n \times n$ matrix $\partial_{x} g\left(z^{*}, 0\right)$ (the Jacobian matrix of the function $g$ with respect to the variable $x$ evaluated at $x=z^{*}$ ) is nonsingular then, as a direct consequence of the Implicit Function Theorem, there exists a unique smooth branch $\chi(\varepsilon)$ of zeros of $g(x, \varepsilon)$ such that $\chi(0)=x^{*}$. However if the matrix $\partial_{x} g\left(x^{*}, 0\right)$ is singular (has non trivial kernel) we have to use the LyapunovSchmidt reduction method to find branches of zeros of $g$ (see, for instance, [8]). Here we generalize some results from $[4,5,14]$, providing a collection of functions $f_{i}, i=1, \ldots, k$, each one called bifurcation function of order $i$, which control the persistence of zeros contained in $\mathcal{Z}$.

The second problem goes back to the works of Malkin [17] and Roseau [18], whose have studied the persistence of periodic solutions for the differential system (2) with $k=1$. Let $x(t, z, \varepsilon)$ denote its solution such that $x(0, z, \varepsilon)=z$. In order to find initial conditions $z \in \mathcal{D}$ such that the solution $x(t, z, \varepsilon)$ is $T$-periodic we may consider the function $g(z, \varepsilon)=z-x(T, z, \varepsilon)$, and then try to use the results previously obtained from the first problem. Indeed, if $\mathcal{Z} \subset \mathcal{D}$ is a submanifold of $T$-periodic solutions of the unperturbed system $x^{\prime}=F_{0}(t, z)$ then $g(z, 0)$ vanishes on $\mathcal{Z}$. When $\operatorname{dim}(\mathcal{Z})=n$ this problem is studied at an arbitrary order of $\varepsilon$, see $[9,11]$, even for nonsmooth systems. When $\operatorname{dim}(\mathcal{Z})<n$, this approach has already been used in [4], up to order 1 , and in [5, 6], up to order 2. In [14] this approach was used up to order 3 relaxing some hypotheses assumed in those previous 3 works. In [10], assuming the same hypotheses of $[4,5,6]$, the authors studied this problem at an arbitrary order of $\varepsilon$. Here, following the ideas from $[11,14]$, we improve the results of [10] relaxing some hypotheses and developing the method in a more general way.

This paper is organized as follows. In section 2 we state our main results: Theorem A, in subsection 2.1, dealing with bifurcation of simple zeros of the equation $g(z, \varepsilon)=0$; and Theorem $\mathrm{B}$, in subsection 2.2, dealing with bifurcation of limit cycles of the differential equation $x^{\prime}=F(t, z, \varepsilon)$. In sections 3 and 4 we prove Theorems A and B, respectively. In section 5 , as an application of Theorem 
B, we study the birth of limit cycles in a 3D polynomial system. Finally, in section 6 , we study the case when the averaged functions have a continuum of zeros. In this last situation we also provide some results about the stability of the limit cycles.

\section{Statements of the main Results}

Before we state our main results we need some preliminary concepts and definitions. Given $p, q$ and $L$ positive integers, $\gamma_{j}=\left(\gamma_{j 1}, \ldots, \gamma_{j p}\right) \in \mathbb{R}^{p}$ for $j=1, \ldots, L$ and $\bar{z} \in \mathbb{R}^{p}$. Let $G: \mathbb{R}^{p} \rightarrow \mathbb{R}^{q}$ be a sufficiently smooth function, then the $L$-th Frechet derivative of $G$ at $\bar{z}$ is denoted by $\partial^{L} G(\bar{z})$, a symmetric $L$-multilinear map, which applied to a "product" of $L p$-dimensional vectors denoted as $\bigodot_{j=1}^{L} \gamma_{j} \in \mathbb{R}^{p L}$ gives

$$
\partial^{L} G(\bar{z}) \bigodot_{j=1}^{L} \gamma_{j}=\sum_{i_{1}, \ldots, i_{L}=1}^{p} \frac{\partial^{L} G(\bar{z})}{\partial b_{i_{1}} \cdots \partial b_{i_{L}}} \gamma_{1 i_{1}} \cdots \gamma_{L i_{L}} .
$$

The above expression is indeed the Gâteaux derivative

$$
\begin{aligned}
\partial^{L} G(\bar{z}) \bigodot_{j=1}^{L} \gamma_{j} & =\left.\frac{\partial}{\partial \tau_{1} \partial \tau_{2} \ldots \partial \tau_{L}} G\left(\bar{z}+\tau_{1} \gamma_{1}+\tau_{2} \gamma_{2}+\cdots+\tau_{L} \gamma_{L}\right)\right|_{\tau_{1}=\cdots=\tau_{L}=0} \\
& =\partial\left(\ldots \partial\left(\partial G(\bar{z}) \gamma_{1}\right) \gamma_{2} \ldots\right) \gamma_{L} .
\end{aligned}
$$

We take $\partial^{0}$ as the identity operator.

2.1. The Lyapunov-Schmidt reduction method. We consider the function

$$
g(z, \varepsilon)=\sum_{i=0}^{k} \varepsilon^{i} g_{i}(z)+\mathcal{O}\left(\varepsilon^{k+1}\right),
$$

where $g_{i}: \mathcal{D} \rightarrow \mathbb{R}^{n}$ is a $\mathcal{C}^{k+1}$ function, $k \geq 1$, for $i=0,1, \ldots, k$, being $\mathcal{D}$ an open bounded subset of $\mathbb{R}^{n}$. For $m<n$, let $V$ be an open bounded subset of $\mathbb{R}^{m}$ and $\beta: \mathrm{Cl}(V) \rightarrow \mathbb{R}^{n-m}$ a $\mathcal{C}^{k+1}$ function, such that

$$
\mathcal{Z}=\left\{z_{\alpha}=(\alpha, \beta(\alpha)): \alpha \in \mathrm{Cl}(V)\right\} \subset \mathcal{D} .
$$

As usual $\mathrm{Cl}(V)$ denotes the closure of the set $V$.

As the main hypothesis we assume that

$\left(\mathrm{H}_{a}\right)$ the function $g_{0}$ vanishes on the $m$-dimensional submanifold $\mathcal{Z}$ of $\mathcal{D}$.

Using the Lyapunov-Schmidt reduction method we shall develop the bifurcation functions of order $i$, for $i=1,2, \ldots, k$, which control, for $|\varepsilon| \neq 0$ small enough, the existence of branches of zeros $z(\varepsilon)$ of (3) bifurcating from $\mathcal{Z}$, that is from $z(0) \in \mathcal{Z}$. With this purpose we introduce some notation. The functions 
$\pi: \mathbb{R}^{m} \times \mathbb{R}^{n-m} \rightarrow \mathbb{R}^{m}$ and $\pi^{\perp}: \mathbb{R}^{m} \times \mathbb{R}^{n-m} \rightarrow \mathbb{R}^{n-m}$ denote the projections onto the first $m$ coordinates and onto the last $n-m$ coordinates, respectively. For a point $z \in \mathcal{D}$ we also consider $z=(a, b) \in \mathbb{R}^{m} \times \mathbb{R}^{n-m}$.

For $i=1,2, \ldots, k$, we define the bifurcation functions $f_{i}: \mathrm{Cl}(V) \rightarrow \mathbb{R}^{m}$ of order $i$ as

$$
f_{i}(\alpha)=\pi g_{i}\left(z_{\alpha}\right)+\sum_{l=1}^{i} \sum_{S_{l}} \frac{1}{c_{1} ! c_{2} ! 2 ! c_{2} \cdots c_{l} ! ! ! c_{l}} \partial_{b}^{L} \pi g_{i-l}\left(z_{\alpha}\right) \bigodot_{j=1}^{l} \gamma_{j}(\alpha)^{c_{j}}, \quad \text { and }
$$

$$
\mathcal{F}^{k}(\alpha, \varepsilon)=\sum_{i=1}^{k} \varepsilon^{i} f_{i}(\alpha)
$$

where $\gamma_{i}: V \rightarrow \mathbb{R}^{n-m}$, for $i=1,2, \ldots, k$, are defined recurrently as

$$
\begin{aligned}
\gamma_{1}(\alpha)= & -\Delta_{\alpha}^{-1} \pi^{\perp} g_{1}\left(z_{\alpha}\right) \text { and } \\
\gamma_{i}(\alpha)= & -i ! \Delta_{\alpha}^{-1}\left(\sum_{S_{i}^{\prime}} \frac{1}{c_{1} ! c_{2} ! 2 ! c_{2} \cdots c_{i-1} !(i-1) ! c_{i-1}} \partial_{b}^{I^{\prime}} \pi^{\perp} g_{0}\left(z_{\alpha}\right) \bigodot_{j=1}^{i-1} \gamma_{j}(\alpha)^{c_{j}}\right. \\
& \left.+\sum_{l=1}^{i-1} \sum_{S_{l}} \frac{1}{c_{1} ! c_{2} ! 2 ! c_{2} \cdots c_{l} ! l ! c_{l}} \partial_{b}^{L} \pi^{\perp} g_{i-l}\left(z_{\alpha}\right) \bigodot_{j=1}^{l} \gamma_{j}(\alpha)^{c_{j}}\right)
\end{aligned}
$$

Here $S_{l}$ is the set of all $l$-tuples of non-negative integers $\left(c_{1}, c_{2}, \cdots, c_{l}\right)$ satisfying $c_{1}+2 c_{2}+\cdots+l c_{l}=l, L=c_{1}+c_{2}+\cdots+c_{l}$, and $S_{i}^{\prime}$ is the set of all $(i-1)$-tuples of non-negative integers satisfying $c_{1}+2 c_{2}+\cdots+(i-1) c_{i-1}=i, I^{\prime}=c_{1}+c_{2}+\cdots+c_{i-1}$ and $\Delta_{\alpha}=\frac{\partial \pi^{\perp} g_{0}}{\partial b}\left(z_{\alpha}\right)$.

We clarify that $S_{0}=S_{0}^{\prime}=\emptyset$, and when $c_{j}=0$, for some $j$, then the term $\gamma_{j}$ does not appear in the "product" $\bigodot_{j=1}^{l} \gamma_{j}(\alpha)^{c_{j}}$.

Recently in [15] the Bell polynomials were used to provide an alternative formula for recurrences of kind (5) and (7). This new formula can make easier the computational implementation of the bifurcation functions (6).

The next theorem is the first main result of this paper. For sake of simplicity we take $f_{0}=0$.

Theorem A. Let $\Delta_{\alpha}$ denote the lower right corner $(n-m) \times(n-m)$ matrix of the Jacobian matrix $D g_{0}\left(z_{\alpha}\right)$. In additional to hypothesis $\left(H_{a}\right)$ we assume that

(i) for each $\alpha \in \mathrm{Cl}(V)$, $\operatorname{det}\left(\Delta_{\alpha}\right) \neq 0$;

(ii) for some $r \in\{1, \ldots, k\}, f_{1}=f_{2}=\cdots=f_{r-1}=0$ and $f_{r}$ is not identically zero; 
(iii) there exists a small parameter $\varepsilon_{0}>0$ such that for each $\varepsilon \in\left[-\varepsilon_{0}, \varepsilon_{0}\right]$ there exists $a_{\varepsilon} \in V$ satisfying $\mathcal{F}^{k}\left(a_{\varepsilon}, \varepsilon\right)=0$;

(iv) there exist a constant $P_{0}>0$ and a positive integer $l \leq(k+r+1) / 2$ such that

$$
\left|\partial_{\alpha} \mathcal{F}^{k}\left(a_{\varepsilon}, \varepsilon\right) \cdot \alpha\right| \geq P_{0}|\varepsilon|^{l}|\alpha|, \quad \text { for } \quad \alpha \in V \text {. }
$$

Then, for $|\varepsilon| \neq 0$ sufficiently small, there exists $z(\varepsilon)$ such that $g(z(\varepsilon), \varepsilon)=0$ with $\left|\pi^{\perp} z(\varepsilon)-\pi^{\perp} z_{a_{\varepsilon}}\right|=\mathcal{O}(\varepsilon)$ and $\left|\pi z(\varepsilon)-\pi z_{a_{\varepsilon}}\right|=\mathcal{O}\left(\varepsilon^{k+1-l}\right)$.

Theorem A is proved in section 3.

In the next corollary we present a classical result in the literature, which is a direct consequence of Theorem A.

Corollary 1. In addiction to hypothesis $\left(H_{a}\right)$ we assume that $f_{1}=f_{2}=\cdots=$ $f_{k-1}=0$, that is $r=k$, and that for each $\alpha \in \mathrm{Cl}(V)$, $\operatorname{det}\left(\Delta_{\alpha}\right) \neq 0$. If there exists $\alpha^{*} \in V$ such that $f_{k}\left(\alpha^{*}\right)=0$ and $\operatorname{det}\left(D f_{k}\left(\alpha^{*}\right)\right) \neq 0$, then there exists a branch of zeros $z(\varepsilon)$ with $g(z(\varepsilon), \varepsilon)=0$ and $\left|z(\varepsilon)-z_{\alpha^{*}}\right|=\mathcal{O}(\varepsilon)$.

Corollary 1 is proved in section 3

2.2. Continuation of periodic solutions. As an application of Theorem A we study higher order bifurcation of periodic solutions of the following $T$-periodic $\mathcal{C}^{k+1}, k \geq 1$, differential system

$$
x^{\prime}=F(t, x, \varepsilon)=F_{0}(t, x)+\sum_{i=1}^{k} \varepsilon^{i} F_{i}(t, x)+\mathcal{O}\left(\varepsilon^{k+1}\right), \quad(t, z) \in \mathbb{S}^{1} \times \mathcal{D} .
$$

Here $\mathbb{S}^{1}=\mathbb{R} /(T \mathbb{Z})$ with $T \mathbb{Z}=\{T, 2 T, \ldots\}$ and the prime denotes derivative with respect to time $t$. Now the manifold $\mathcal{Z}$, defined in (4), is seen as a set of initial conditions of the unperturbed system

$$
x^{\prime}(t)=F_{0}(t, x) .
$$

In fact we shall assume that all solutions of the unperturbed system starting at points of $\mathcal{Z}$ are $T$-periodic, recall that the dimension of $\mathcal{Z}$ is $m \leq n$. Formally, let $x(\cdot, z, 0):\left[0, t_{z}\right) \rightarrow \mathbb{R}^{n}$ denote the solution of $(9)$ such that $x(0, z, 0)=z$, we assume that

$\left(\mathrm{H}_{b}\right) \mathcal{Z} \subset \mathcal{D}$, for each $\alpha \in \mathrm{Cl}(V)$ the unique solution $x\left(t, z_{\alpha}, 0\right)$ of $(9)$ is $T$ periodic.

As usual $x(\cdot, z, \varepsilon):\left[0, t_{(z, \varepsilon)}\right) \rightarrow \mathbb{R}^{n}$ denotes a solution of system (8) such that $x(0, z, \varepsilon)=z$. Moreover, let $Y(t, z)$ be a fundamental matrix solution of the linear differential system

$$
y^{\prime}=\frac{\partial}{\partial x} F_{0}(t, x(t, z, 0)) y
$$


For sake of simplicity when $z=z_{\alpha} \in \mathcal{Z}$ we denote $Y_{\alpha}(t)=Y\left(t, z_{\alpha}\right)$.

Given fundamental matrix solution $Y(t, z)$, the averaged functions of order $i$, $g_{i}: \mathrm{Cl}(V) \rightarrow \mathbb{R}^{n}, i=1,2, \ldots, k$, of system (8) is defined as

$$
g_{i}(z)=Y(T, z)^{-1} \frac{y_{i}(T, z)}{i !},
$$

where

$$
\begin{aligned}
y_{1}(t, z)= & Y(t, z) \int_{0}^{t} Y(s, z)^{-1} F_{1}(s, x(s, z, 0)) d s \\
y_{i}(t, z)= & i ! Y(t, z) \int_{0}^{t} Y(s, z)^{-1}\left(F_{i}(s, x(s, z, 0))\right. \\
& +\sum_{S_{i}^{\prime}} \frac{1}{b_{1} ! b_{2} ! 2 ! b_{2} \cdots b_{i-1} !(i-1) ! b^{b_{i-1}}} \partial^{I^{\prime}} F_{0}(s, x(s, z, 0)) \bigodot_{j=1}^{i-1} y_{j}(s, z)^{b_{j}} \\
& \left.+\sum_{l=1}^{i-1} \sum_{S_{l}} \frac{1}{b_{1} ! b_{2} ! 2 ! b_{2} \cdots b_{l} ! l ! b_{l}} \partial^{L} F_{i-l}(s, x(s, z, 0)) \bigodot_{j=1}^{l} y_{j}(s, z)^{b_{j}}\right) d s .
\end{aligned}
$$

Using now the functions $g_{i}$ as stated in (11) we define the functions $f_{i}, \mathcal{F}^{k}$, and $\gamma_{i}$ given by (5), (6), and (7), respectively.

Recently in [15] the Bell polynomials were used to provide an alternative formula for the recurrence (12). This new formula can also make easier the computational implementation of the bifurcation functions (11).

The next theorem is the second main result of this paper. Again, for sake of simplicity, we assume that $f_{0}=0$.

Theorem B. Let $\Delta_{\alpha}$ denote the lower right corner $(n-m) \times(n-m)$ matrix of the matrix $Y(0, z)^{-1}-Y(T, z)^{-1}$. In additional to hypothesis $\left(H_{b}\right)$ we assume that

(i) for each $\alpha \in \mathrm{Cl}(V)$, $\operatorname{det}\left(\Delta_{\alpha}\right) \neq 0$;

(ii) for some $r \in\{1, \ldots, k\}, f_{1}=f_{2}=\cdots=f_{r-1}=0$ and $f_{r}$ is not identically zero;

(iii) there exists a small parameter $\varepsilon_{0}>0$ such that for each $\varepsilon \in\left[-\varepsilon_{0}, \varepsilon_{0}\right]$ there exists $a_{\varepsilon} \in V$ satisfying $\mathcal{F}^{k}\left(a_{\varepsilon}, \varepsilon\right)=0$;

(iv) there exist a constant $P_{0}>0$ and a positive integer $l \leq(k+r+1) / 2$ such that

$$
\left|\partial_{\alpha} \mathcal{F}^{k}\left(a_{\varepsilon}, \varepsilon\right) \cdot \alpha\right| \geq P_{0}\left|\varepsilon^{l}\right||\alpha|, \quad \text { for } \quad \alpha \in V .
$$

Then, for $|\varepsilon| \neq 0$ sufficiently small, there exists a T-periodic solution $\varphi(t, \varepsilon)$ of system (8) such that $\left|\pi \varphi(0, \varepsilon)-\pi z_{a_{\varepsilon}}\right|=\mathcal{O}\left(\varepsilon^{k+1-l}\right)$, and $\left|\pi^{\perp} \varphi(0, \varepsilon)-\pi^{\perp} z_{a_{\varepsilon}}\right|=$ $\mathcal{O}(\varepsilon)$. 
Theorem B is proved in section 4 .

In the next corollary we present a classical result in the literature, which is a direct consequence of Theorem B.

Corollary 2. In addiction to hypothesis $\left(H_{b}\right)$ we assume that $f_{1}=f_{2}=\cdots=$ $f_{k-1}=0, r=k$ and that for each $\alpha \in \mathrm{Cl}(V)$, $\operatorname{det}\left(\Delta_{\alpha}\right) \neq 0$. If there exists $\alpha^{*} \in V$ such that $f_{k}\left(\alpha^{*}\right)=0$ and $\operatorname{det}\left(D f_{k}\left(\alpha^{*}\right)\right) \neq 0$, then there exists a $T$ periodic solution $\varphi(t, \varepsilon)$ of (8) such that $\left|\varphi(0, \varepsilon)-z_{\alpha^{*}}\right|=\mathcal{O}(\varepsilon)$.

Corollary 2 is proved in section 4. An application of Theorem B is performed in Section 5.

It is worth to emphasize that Theorem $\mathrm{B}$ is still true when $m=n$. In fact, assuming that $V$ is an open subset of $\mathbb{R}^{n}$ then $\mathcal{Z}=\mathrm{Cl}(V) \subset \mathcal{D}$ and the projections $\pi$ and $\pi^{\perp}$ become the identity and the null operator respectively. Moreover, in this case the bifurcation functions $f_{i}: V \rightarrow \mathbb{R}^{n}$, for $i=1,2, \ldots, k$, are the averaged functions $f_{i}(\alpha)=g_{i}(\alpha)$ defined in (11). Thus we have the following corollary, which recover the main result from [11].

Corollary 3. Consider $m=n, z_{\alpha}=\alpha \in \mathcal{Z}$ and the hypothesis $\left(H_{b}\right)$. Thus the result of Theorem $B$ holds without any assumption about $\Delta_{\alpha}$.

\section{Proof of Theorem A and Corollary 1}

A useful tool to study the zeros of a function is the Browder degree (see the Appendix 6.2 for some of their properties). Let $g \in C^{1}(\mathcal{D}), \operatorname{Cl}(V) \subset \mathcal{D}$ and $\mathbf{Z}_{g}=\{z \in V: g(z)=0\}$. We also assume that $J_{g}(z) \neq 0$ for all $z \in \mathbf{Z}_{g}$, where $J_{g}(z)$ is the Jacobian determinant of $g$ at $z$. This assures that the set $\mathbf{Z}_{g}$ is formed by a finite number of isolated points. Then the Brouwer degree of $g$ at 0 is

$$
d_{B}(g, V, 0)=\sum_{z \in \mathbf{Z}_{g}} \operatorname{sign}\left(J_{g}(z)\right)
$$

As one of the main properties of the Brouwer degree we have that: "if $d(f, V, 0) \neq$ 0 then there exists $x_{0} \in V$ such that $f\left(x_{0}\right)=0$ " (see item (i) of Theorem 8 from Appendix 6.2).

The next result is a key lemma for proving Theorem A.

Lemma 4. Let $V$ be an open bounded subset of $\mathbb{R}^{m}$. Consider the continuous functions $f_{i}: \mathrm{Cl}(V) \rightarrow \mathbb{R}^{n}, i=0,1, \cdots, \kappa$, and $f, g, r: \mathrm{Cl}(V) \times\left[-\varepsilon_{0}, \varepsilon_{0}\right] \rightarrow \mathbb{R}^{n}$ given by

$$
g(x, \varepsilon)=f_{0}(x)+\varepsilon f_{1}(x)+\cdots+\varepsilon^{\kappa} f_{\kappa}(x) \text { and } f(x, \varepsilon)=g(x, \varepsilon)+\varepsilon^{\kappa+1} r(x, \varepsilon) .
$$

Let $V_{\varepsilon} \subset V, R=\max \left\{|r(x, \varepsilon)|:(x, \varepsilon) \in \mathrm{Cl}(V) \times\left[-\varepsilon_{0}, \varepsilon_{0}\right]\right\}$ and assume that $|g(x, \varepsilon)|>R|\varepsilon|^{\kappa+1}$ for all $x \in \partial V_{\varepsilon}$ and $\varepsilon \in\left[-\varepsilon_{0}, \varepsilon_{0}\right] \backslash\{0\}$. Then for each $\varepsilon \in$ $\left[-\varepsilon_{0}, \varepsilon_{0}\right] \backslash\{0\}$ we have $d_{B}\left(f(\cdot, \varepsilon), V_{\varepsilon}, 0\right)=d_{B}\left(g(\cdot, \varepsilon), V_{\varepsilon}, 0\right)$. 
Proof. For a fixed $\varepsilon \in\left[-\varepsilon_{0}, \varepsilon_{0}\right] \backslash\{0\}$, consider a continuous homotopy between $g(\cdot, \varepsilon)$ and $f(\cdot, \varepsilon)$ given by $g_{t}(x, \varepsilon)=g(x, \varepsilon)+t(f(x, \varepsilon)-g(x, \varepsilon))=g(x, \varepsilon)+$ $t \varepsilon^{\kappa+1} r(x, \varepsilon)$. We claim that $0 \notin g_{t}\left(\partial V_{\varepsilon}, \varepsilon\right)$ for every $t \in[0,1]$. As usual $\partial V_{\varepsilon}$ denotes the boundary of the set $V_{\varepsilon}$. Indeed, assuming that $0 \in g_{t_{\varepsilon}}\left(\partial V_{\varepsilon}, \varepsilon\right)$, for some $t_{\varepsilon} \in[0,1]$, we may find $x_{\varepsilon} \in \partial V_{\varepsilon}$ such that $g_{t_{\varepsilon}}\left(x_{\varepsilon}, \varepsilon\right)=0$ and, consequently, $g\left(x_{\varepsilon}, \varepsilon\right)=-t_{\varepsilon} \varepsilon^{\kappa+1} r\left(x_{\varepsilon}, \varepsilon\right)$. Thus $\left|g\left(x_{\varepsilon}, \varepsilon\right)\right| \leq R|\varepsilon|^{\kappa+1}$, which contradicts the hypothesis $\left|g\left(x_{\varepsilon}, \varepsilon\right)\right|>R|\varepsilon|^{\kappa+1}$. From Theorem (8) (iii) we conclude that $d_{B}\left(g_{t}(\cdot, \varepsilon), V_{\varepsilon}, 0\right)$ is constant for $t \in[0,1]$ and then $d_{B}\left(f(\cdot, \varepsilon), V_{\varepsilon}, 0\right)=$ $d_{B}\left(g(\cdot, \varepsilon), V_{\varepsilon}, 0\right)$.

The above lemma provides a stratagem to track zeros of the perturbed function $f(x, \varepsilon)$ using a shrinking neighborhood around the zeros of $g(x, \varepsilon)$ that preserves its Brouwer degree. The way how it works can be blurry at the first moment, so to make it clear we present the following example:

Example 1. Consider the real function $f(x, \varepsilon)=g(x, \varepsilon)+\varepsilon^{2} r(x, \varepsilon)$ with $(x, \varepsilon) \in$ $[-1,1] \times\left[-\varepsilon_{0}, \varepsilon_{0}\right], g(x, \varepsilon)=x^{2}-\varepsilon x$, and $|r(x, \varepsilon)| \leq 1 / 5$. The function $g(x, \varepsilon)$ has two zeros $a=0$ and $a_{\varepsilon}=\varepsilon$. Taking $V_{\varepsilon}=(\varepsilon / 2,3 \varepsilon / 2)$ we have that, for $|\varepsilon| \neq 0$ sufficiently small, $a_{\varepsilon} \in V_{\varepsilon}$ and $d_{B}\left(g(\cdot, \varepsilon), V_{\varepsilon}, 0\right)=1$ (see Definition (13)). Furthermore $\partial V_{\varepsilon}=\{\varepsilon / 2,3 \varepsilon / 2\},|g(\varepsilon / 2, \varepsilon)|=\varepsilon^{2} / 4$, and $|g(3 \varepsilon / 2, \varepsilon)|=3 \varepsilon^{2} / 4$. Thus $|g(x, \varepsilon)|>\varepsilon^{2} / 5 \geq \varepsilon^{2} \max \left\{|r(x, \varepsilon)|:(x, \varepsilon) \in[0,1] \times\left[-\varepsilon_{0}, \varepsilon_{0}\right]\right\}$. Therefore from the previous lemma we know that $d_{B}\left(f(\cdot, \varepsilon), V_{\varepsilon}, 0\right)=1$. From the above property of the Brouwer degree we conclude that there exists $\alpha_{\varepsilon} \in V_{\varepsilon}$ such that $f\left(\alpha_{\varepsilon}, \varepsilon\right)=0$.

Now we recall the Faá di Bruno's Formula (see [12]) about the $l^{\text {th }}$ derivative of a composite function.

Faá di Bruno's Formula If $u$ and $v$ are functions with a sufficient number of derivatives, then

$$
\frac{d^{l}}{d t^{l}} u(v(t))=\sum_{S_{l}} \frac{l !}{b_{1} ! b_{2} ! 2 !^{b_{2}} \cdots b_{l} ! l ! !_{l}} u^{(L)}(v(t)) \bigodot_{j=1}^{l} v^{(j)}(t)^{b_{j}},
$$

where $S_{l}$ is the set of all l-tuples of non-negative integers $\left(b_{1}, b_{2}, \cdots, b_{l}\right)$ which are solutions of the equation $b_{1}+2 b_{2}+\cdots+l b_{l}=l$ and $L=b_{1}+b_{2}+\cdots+b_{l}$.

The remainder of this section consists in the proof of Theorem A, which is split in several claims, and the proof Corollary 1

Proof of Theorem A. We consider $g=\left(\pi g, \pi^{\perp} g\right), g_{i}=\left(\pi g_{i}, \pi^{\perp} g_{i}\right)$ for $i=0,1,2, \ldots, k$, and $z=(a, b) \in \mathbb{R}^{m} \times \mathbb{R}^{n-m}$ for $z \in \mathcal{D}$. So

$$
\frac{\partial g}{\partial z}\left(z_{\alpha}, 0\right)=D g_{0}\left(z_{\alpha}\right)=\left(\begin{array}{cc}
\frac{\partial \pi g_{0}}{\partial a}\left(z_{\alpha}\right) & \frac{\partial \pi g_{0}}{\partial b}\left(z_{\alpha}\right) \\
\frac{\partial \pi^{\perp} g_{0}}{\partial a}\left(z_{\alpha}\right) & \frac{\partial \pi^{\perp} g_{0}}{\partial b}\left(z_{\alpha}\right)
\end{array}\right)
$$


We write $\Delta_{\alpha}=\frac{\partial \pi^{\perp} g_{0}}{\partial b}\left(z_{\alpha}\right)$. From hypotheses, $\pi^{\perp} g(\alpha, \beta(\alpha), 0)=\pi^{\perp} g_{0}\left(z_{\alpha}\right)=0$ and

$$
\operatorname{det}\left(\frac{\partial \pi^{\perp} g}{\partial b}(\alpha, \beta(\alpha), 0)\right)=\operatorname{det}\left(\frac{\partial \pi^{\perp} g_{0}}{\partial b}\left(z_{\alpha}\right)\right)=\operatorname{det}\left(\Delta_{\alpha}\right) \neq 0 .
$$

Thus applying the Implicit Function Theorem it follows that there exists an open neighborhood $U \times\left(-\varepsilon_{1}, \varepsilon_{1}\right)$ of $\mathrm{Cl}(V) \times\{0\}$ with $\varepsilon_{1} \leq \varepsilon_{0}$, and a $\mathcal{C}^{k+1}$ function $\bar{\beta}: U \times\left(-\varepsilon_{1}, \varepsilon_{1}\right) \rightarrow \mathbb{R}^{n-m}$ such that $\pi^{\perp} g(a, \bar{\beta}(a, \varepsilon), \varepsilon)=0$ for each $(a, \varepsilon) \in$ $U \times\left(-\varepsilon_{1}, \varepsilon_{1}\right)$ and $\bar{\beta}(\alpha, 0)=\beta(\alpha)$ for every $\alpha \in \mathrm{Cl}(V)$.

From here, this proof will be split in several claims.

Claim 1. The equality $\left(\partial^{i} \bar{\beta} / \partial \varepsilon^{i}\right)(\alpha, 0)=\gamma_{i}(\alpha)$ holds for $i=1,2, \ldots, k$.

Firstly, it is easy to check that $(\partial \bar{\beta} / \partial \varepsilon)(\alpha, 0)=\gamma_{1}(\alpha)$. Now, for some fixed $i \in\{1,2, \ldots, k\}$, we assume by induction hypothesis that $\left(\partial^{s} \bar{\beta} / \partial \varepsilon^{s}\right)(\alpha, 0)=\gamma_{s}(\alpha)$ for $s=1, \ldots, i-1$. In what follows we prove the claim for $s=i$. Consider

$$
\pi^{\perp} g(\alpha, \bar{\beta}(\alpha, \varepsilon), \varepsilon)=\sum_{i=0}^{k} \varepsilon^{i} \pi^{\perp} g_{i}(\alpha, \bar{\beta}(\alpha, \varepsilon))+\mathcal{O}\left(\varepsilon^{k+1}\right)=0 .
$$

Expanding each function $\varepsilon \mapsto \pi^{\perp} g_{i}(\alpha, \bar{\beta}(\alpha, \varepsilon))$ in Taylor series we obtain

$$
\begin{aligned}
\pi^{\perp} g(\alpha, \bar{\beta}(\alpha, \varepsilon), \varepsilon)= & \sum_{i=0}^{k}\left(\left.\varepsilon^{i} \sum_{l=0}^{i} \frac{1}{l !} \frac{\partial^{l}}{\partial \varepsilon^{l}} \pi^{\perp} g_{i-l}(\alpha, \bar{\beta}(\alpha, \varepsilon))\right|_{\varepsilon=0}\right) \\
& +\mathcal{O}\left(\varepsilon^{k+1}\right)=0 .
\end{aligned}
$$

Applying the the Faà di Bruno's formula we obtain

$$
\begin{aligned}
\left.\frac{\partial^{l}}{\partial \varepsilon^{l}} \pi^{\perp} g_{i-l}(\alpha, \bar{\beta}(\alpha, \varepsilon))\right|_{\varepsilon=0}= & \sum_{S_{l}}\left(\frac{l !}{b_{1} ! b_{2} ! 2 !^{b_{2}} \cdots b_{l} ! l ! !_{l} b_{b}} \partial_{b}^{L} \pi^{\perp} g_{i-l}(\alpha, \bar{\beta}(\alpha, 0))\right. \\
& \left.\bigodot_{j=1}^{l} \frac{\partial^{j}}{\partial \varepsilon^{j}} \bar{\beta}(\alpha, 0)^{b_{j}}\right) .
\end{aligned}
$$

Substituting (15) in (14) we get

$$
\begin{aligned}
\pi^{\perp} g(\alpha, \bar{\beta}(\alpha, \varepsilon), \varepsilon)= & \sum_{i=0}^{k} \varepsilon^{i}\left(\sum_{l=0}^{i} \sum_{S_{l}} \frac{1}{b_{1} ! b_{2} ! 2 ! b_{2} \cdots b_{l} ! l ! b_{l}} \partial_{b}^{L} \pi^{\perp} g_{i-l}(\alpha, \bar{\beta}(\alpha, 0))\right. \\
& \left.\bigodot_{j=1}^{l} \frac{\partial^{j}}{\partial \varepsilon^{j}} \bar{\beta}(\alpha, 0)^{b_{j}}\right)+\mathcal{O}\left(\varepsilon^{k+1}\right)=0 .
\end{aligned}
$$


Since the previous equation is equal to zero for $|\varepsilon|$ sufficiently small, the coefficients of each power of $\varepsilon$ vanish. Then for $0 \leq i \leq k$ and $(\alpha, \varepsilon) \in U \times\left(-\varepsilon_{1}, \varepsilon_{1}\right)$ we have

$$
\sum_{l=0}^{i} \sum_{S_{l}} \frac{1}{b_{1} ! b_{2} ! 2 ! b_{2} \cdots b_{l} ! l ! b_{l}} \partial_{b}^{L} \pi^{\perp} g_{i-l}(\alpha, \bar{\beta}(\alpha, 0)) \bigodot_{j=1}^{l} \frac{\partial^{j}}{\partial \varepsilon^{j}} \bar{\beta}(\alpha, 0)^{b_{j}}=0 .
$$

This equation can be rewritten as

$$
\begin{aligned}
0= & \sum_{l=0}^{i-1} \sum_{S_{l}} \frac{1}{b_{1} ! b_{2} ! 2 !^{b_{2}} \cdots b_{l} ! l ! b_{l}} \partial_{b}^{L} \pi^{\perp} g_{i-l}(\alpha, \bar{\beta}(\alpha, 0)) \bigodot_{j=1}^{l} \frac{\partial^{j}}{\partial \varepsilon^{j}} \bar{\beta}(\alpha, 0)^{b_{j}} \\
& +\sum_{S_{i}^{\prime}} \frac{1}{b_{1} ! b_{2} ! 2 !^{b_{2}} \cdots b_{i-1} !(i-1) !^{b_{i-1}}} \partial_{b}^{I^{\prime}} \pi^{\perp} g_{0}(\alpha, \bar{\beta}(\alpha, 0)) \bigodot_{j=1}^{i-1} \frac{\partial^{j}}{\partial \varepsilon^{j}} \bar{\beta}(\alpha, 0)^{b_{j}} \\
& +\frac{1}{i !} \partial_{b} \pi^{\perp} g_{0}(\alpha, \bar{\beta}(\alpha, 0)) \frac{\partial^{i}}{\partial \varepsilon^{i}} \bar{\beta}(\alpha, 0) .
\end{aligned}
$$

Here $S_{i}^{\prime}$ is the set of all $(i-1)$-tuples of non-negative integers satisfying $b_{1}+$ $2 b_{2}+\cdots+(i-1) b_{i-1}=i, I^{\prime}=b_{1}+b_{2}+\cdots+b_{i-1}$. Finally, using the induction hypothesis, equation (16) becomes

$$
\begin{aligned}
\frac{\partial^{i} \beta}{\partial \varepsilon^{i}}(\alpha, 0) & =-i ! \Delta_{\alpha}^{-1}\left(\sum_{S_{i}^{\prime}} \frac{1}{b_{1} ! b_{2} ! 2 ! b_{2} \cdots b_{(i-1)} !(i-1) ! b_{i-1}} \partial_{b}^{I^{\prime}} \pi^{\perp} g_{0}\left(z_{\alpha}\right) \bigodot_{j=1}^{i-1} \gamma_{j}(\alpha)^{b_{s}}\right. \\
& \left.+\sum_{l=0}^{i-1} \sum_{S_{l}} \frac{1}{b_{1} ! b_{2} ! 2 ! b_{2} \cdots b_{l} ! ! !^{b_{l}}} \partial_{b}^{L} \pi^{\perp} g_{i-l}\left(z_{\alpha}\right) \bigodot_{j=1}^{l} \gamma_{j}(\alpha)^{b_{s}}\right)=\gamma_{i}(\alpha) .
\end{aligned}
$$

This concludes the proof of Claim 1.

Claim 2. Let $\delta: U \times\left(-\varepsilon_{1}, \varepsilon_{1}\right) \rightarrow \mathbb{R}^{m}$ be the $C^{k+1}$ function defined as $\delta(\alpha, \varepsilon)=$ $\pi g(\alpha, \bar{\beta}(\alpha, \varepsilon), \varepsilon)$. Then the equality $\left(\partial^{i} \delta / \partial \varepsilon^{i}\right)(\alpha, 0)=i ! f_{i}(\alpha)$ holds for $i=1,2, \ldots, k$.

From (3) the function $\delta$ reads

$$
\delta(\alpha, \varepsilon)=\sum_{j=0}^{k} \varepsilon^{j} \pi g_{j}(\alpha, \bar{\beta}(\alpha, \varepsilon))+\mathcal{O}\left(\varepsilon^{k+1}\right) .
$$

So computing its $i$ th-derivative, $0 \leq i \leq k$, in the variable $\varepsilon$, we get

$$
\frac{\partial^{i} \delta}{\partial \varepsilon^{i}}(\alpha, \varepsilon)=\sum_{j=0}^{i} \sum_{q=0}^{i}\left(\begin{array}{l}
i \\
q
\end{array}\right)\left(\varepsilon^{j}\right)^{(i-q)} \frac{\partial^{q} \pi g_{j}}{\partial \varepsilon^{q}}(\alpha, \bar{\beta}(\alpha, \varepsilon))+\mathcal{O}(\varepsilon) .
$$


Taking $\varepsilon=0$ and $l=i-j$ we obtain

$$
\frac{\partial^{i} \delta}{\partial \varepsilon^{i}}(\alpha, 0)=\left.\sum_{l=1}^{i} \frac{i !}{l !} \frac{\partial^{l} \pi g_{i-l}}{\partial \varepsilon^{l}}(\alpha, \bar{\beta}(\alpha, \varepsilon))\right|_{\varepsilon=0}+i ! \pi g_{i}\left(z_{\alpha}\right) .
$$

Finally using the Faà di Brunno's formula and Claim 1 we have

$$
\begin{aligned}
\frac{\partial^{i} \delta}{\partial \varepsilon^{i}}(\alpha, 0) & =\sum_{l=1}^{i} \frac{i !}{l !} \sum_{S_{l}} \frac{l !}{c_{1} ! c_{2} ! 2 ! c_{2} \ldots c_{l} ! l ! c_{l}} \partial_{b}^{L} \pi g_{i-l}\left(z_{\alpha}\right) \bigodot_{s=1}^{l} \gamma_{s}(\alpha)^{c_{s}}+i ! \pi g_{i}\left(z_{\alpha}\right) \\
& =i ! f_{i}(\alpha) .
\end{aligned}
$$

This concludes the proof of Claim 2.

Using Claim 2 the function $\delta(\alpha, \varepsilon)$ can be expanded in power series of $\varepsilon$ as

$$
\delta(\alpha, \varepsilon)=\sum_{i=0}^{k} \frac{\varepsilon^{i}}{i !} \frac{\partial^{i} \delta}{\partial \varepsilon^{i}}(\alpha, 0)+\mathcal{O}\left(\varepsilon^{k+1}\right)=\mathcal{F}^{k}(\alpha, \varepsilon)+\mathcal{O}\left(\varepsilon^{k+1}\right),
$$

and, from hypothesis $(i i)$, we have

$$
\widetilde{\delta}(\alpha, \varepsilon):=\frac{\delta(\alpha, \varepsilon)}{\varepsilon^{r}}=\mathcal{G}^{k}(\alpha, \varepsilon)+\mathcal{O}\left(\varepsilon^{k-r+1}\right),
$$

where $\mathcal{G}^{k}(\alpha, \varepsilon)=f_{r}(\alpha)+\varepsilon f_{r+1}(\alpha)+\ldots+\varepsilon^{k-r} f_{k}(\alpha)$. Obviously the equations $\delta(\alpha, \varepsilon)=0$ and $\widetilde{\delta}(\alpha, \varepsilon)=0$ are equivalent for $\varepsilon \neq 0$.

Denote $R\left(\varepsilon_{0}\right)=\max \left\{\left|\widetilde{\delta}(\alpha, \varepsilon)-\mathcal{G}^{k}(\alpha, \varepsilon)\right|:(\alpha, \varepsilon) \in \mathrm{Cl}(V) \times\left[-\varepsilon_{0}, \varepsilon_{0}\right]\right\}$. From the continuity of the functions $\widetilde{\delta}$ and $\mathcal{G}^{k}$ and from the compactness of the set $\mathrm{Cl}(V) \times\left[-\varepsilon_{0}, \varepsilon_{0}\right]$ we know that $R\left(\varepsilon_{0}\right)<\infty$ and $R(0)=0$. In order to study the zeros of $\widetilde{\delta}(\alpha, \varepsilon)$ we use Lemma 4 , for proving the following claim.

Claim 3. Consider $a_{\varepsilon} \in V$ as given in hypothesis (iii) and $\varepsilon \in\left[-\varepsilon_{0}, \varepsilon_{0}\right]$. Then, there exist $\varepsilon_{0}>0$ sufficiently small and, for each $\varepsilon \in\left[-\varepsilon_{0}, \varepsilon_{0}\right]$, a neighborhood $V_{\varepsilon} \subset V$ of $a_{\varepsilon}$ such that $\left|\mathcal{G}^{k}(\bar{\alpha}, \varepsilon)\right|>R\left(\varepsilon_{0}\right)\left|\varepsilon^{k-r+1}\right|$ for all $\bar{\alpha} \in \partial V_{\varepsilon}$. Moreover $V_{\varepsilon}=B\left(a_{\varepsilon}, Q|\varepsilon|^{k+1-l}\right)$ for some $Q>0$.

The parameter $\varepsilon_{0}>0$ will be chosen later on. Given $\varepsilon \in\left[-\varepsilon_{0}, \varepsilon_{0}\right]$, since $\mathcal{G}^{k}(\alpha, \varepsilon)$ is a $\mathcal{C}^{k+1}$ function, $k \geq 1$, we have that

$$
\mathcal{G}^{k}\left(a_{\varepsilon}+h, \varepsilon\right)=\partial_{\alpha} \mathcal{G}^{k}\left(a_{\varepsilon}, \varepsilon\right) h+\rho(h), \quad \rho(h)=\mathcal{O}\left(|h|^{2}\right),
$$

for every $h \in \mathbb{R}^{m}$ such that $\left[a_{\varepsilon}, a_{\varepsilon}+h\right] \subset V$. Moreover, hypotheses $(i i)$ and $(i v)$ imply that

$$
\left|\partial_{\alpha} \mathcal{G}^{k}\left(a_{\varepsilon}, \varepsilon\right) \cdot \alpha\right| \geq P_{0}|\varepsilon|^{l-r}|\alpha| \quad \text { for } \quad \alpha \in V .
$$

Combining expressions (18) and (19) we obtain the following inequality:

$$
\left|\mathcal{G}^{k}\left(a_{\varepsilon}+h, \varepsilon\right)\right| \geq\left(P_{0}-|\varepsilon|^{r-l} \frac{|\rho(h)|}{|h|}\right)|\varepsilon|^{l-r}|h| .
$$


Take $V_{\varepsilon}=B\left(a_{\varepsilon}, Q|\varepsilon|^{k+1-l}\right) \subset V$. A point $\bar{\alpha}_{\varepsilon} \in \partial V_{\varepsilon}$ reads $\bar{\alpha}_{\varepsilon}=a_{\varepsilon}+h_{\varepsilon}$, where $h_{\varepsilon}=u Q|\varepsilon|^{k+1-l} \in \mathbb{R}^{m}$ and $|u|=1$. Moreover, since $\rho(h)=\mathcal{O}\left(|h|^{2}\right)$ we get

$$
|\varepsilon|^{r-l} \frac{\left|\rho\left(h_{\varepsilon}\right)\right|}{\left|h_{\varepsilon}\right|}=|\varepsilon|^{r-l} \mathcal{O}\left(Q|\varepsilon|^{k+1-l}\right)=\mathcal{O}\left(Q|\varepsilon|^{k+r+1-2 l}\right) .
$$

From hypothesis $(i i i), k+r+1-2 l \geq 0$. So, in particular, $\mathcal{O}\left(Q|\varepsilon|^{k+r+1-2 l}\right)=$ $\mathcal{O}(Q)$. Thus, from definition of the symbol $\mathcal{O}$, there exists $c_{0}>0$, which does not depend on $\varepsilon$ and $Q$, such that $|\varepsilon|^{r-l}\left|\rho\left(h_{\varepsilon}\right)\right| /\left|h_{\varepsilon}\right| \leq c_{0} Q$. So the inequality (20) reads

$$
\left|\mathcal{G}^{k}\left(a_{\varepsilon}+h_{\varepsilon}, \varepsilon\right)\right| \geq\left(P_{0}-Q c_{0}\right) Q|\varepsilon|^{k-r+1} .
$$

Note that the polynomial $\mathcal{P}(Q)=\left(P_{0}-Q c_{0}\right) Q$ is positive for $0<Q<P_{0} / c_{0}$ and reach its maximum at $Q^{*}=P_{0} /\left(2 c_{0}\right)$. Moreover $\mathcal{P}\left(Q^{*}\right)=P_{0}^{2} /\left(4 c_{0}\right)$. Since $R(0)=0$, there exists $\varepsilon_{0}>0$ small enough in order that $R\left(\varepsilon_{0}\right)<P_{0}^{2} /\left(4 c_{0}\right)=$ $\mathcal{P}\left(Q^{*}\right)$. Accordingly, taking $Q=Q^{*}$ it follows that $\left|\mathcal{G}^{k}(\bar{\alpha}, \varepsilon)\right|>R\left(\varepsilon_{0}\right)\left|\varepsilon^{k-r+1}\right|$ for all $\bar{\alpha} \in \partial V_{\varepsilon}$ and $\varepsilon \in\left[-\varepsilon_{0}, \varepsilon_{0}\right]$. This concludes the proof of Claim.

Applying Lemma 4 for $g=\widetilde{\delta}$, as defined in (17), $\kappa=k-r$, and $V_{\varepsilon}=$ $B\left(a_{\varepsilon}, Q|\varepsilon|^{k+1-l}\right)$ we conclude that $d_{B}\left(\widetilde{\delta}(\cdot, \varepsilon), V_{\varepsilon}, 0\right)=d_{B}\left(\mathcal{G}^{k}(\cdot, \varepsilon), V_{\varepsilon}, 0\right) \neq 0$. Finally, denoting $z(\varepsilon)=(\alpha(\varepsilon), \bar{\beta}(\alpha(\varepsilon), \varepsilon))$ it follows that $g(z(\varepsilon), \varepsilon)=0$.

Moreover, let $z_{a_{\varepsilon}}=\left(a_{\varepsilon}, \beta\left(a_{\varepsilon}\right)\right)$, then $\left|\pi z(\varepsilon)-\pi z_{a_{\varepsilon}}\right|=\left|\alpha(\varepsilon)-a_{\varepsilon}\right|=\mathcal{O}\left(\varepsilon^{k+1-l}\right)$ and, since $\bar{\beta}$ is Lipschtiz,

$$
\left|\pi^{\perp} z(\varepsilon)-\pi^{\perp} z_{a_{\varepsilon}}\right|=\left|\bar{\beta}(\alpha(\varepsilon), \varepsilon)-\bar{\beta}\left(a_{\varepsilon}, 0\right)\right| \leq L\left|(\alpha(\varepsilon), \varepsilon)-\left(a_{\varepsilon}, 0\right)\right|=\mathcal{O}(\varepsilon) .
$$

This concludes the proof of Theorem A.

Proof of Corollary 1. The basic idea of the proof is to show that $\mathcal{F}^{k}(\alpha)$ satisfies all the hypotheses of Theorem A. From hypotheses, $\mathcal{F}^{k}(\alpha, \varepsilon)=\varepsilon^{k} f_{k}(\alpha)$ and $D f_{k}\left(\alpha^{*}\right)=\varepsilon^{-k} \partial_{\alpha} \mathcal{F}^{k}\left(\alpha^{*}, \varepsilon\right)$ is a homeomorphism on $\mathbb{R}^{n}$. Thus there exist constants $b, c>0$ such that

$$
b|\alpha|<\left|J f_{k}\left(\alpha^{*}\right) \cdot \alpha\right|=\left|\frac{1}{\varepsilon^{k}} \partial_{\alpha} \mathcal{F}^{k}\left(\alpha^{*}, \varepsilon\right) \cdot \alpha\right|<c|\alpha|,
$$

for all $\alpha \in \mathbb{R}^{m}$. Therefore $b\left|\varepsilon^{k}\right||\alpha|<\left|\partial_{\alpha} \mathcal{F}^{k}\left(\alpha^{*}, \varepsilon\right) . \alpha\right|<c\left|\varepsilon^{k}\right||\alpha|$, which implies that $\mathcal{F}^{k}\left(\alpha^{*}\right)$ satisfies hypothesis (iii) of Theorem A, with $l=r=k$. Indeed $(k+r+1) / 2=k+1 / 2>k=l$. Hence the proof follows directly from Theorem A.

\section{Proof of Theorem B and Collorary 2}

The next result is needed in the proof of Theorem B. 
Lemma 5 (Fundamental Lemma). Let $x(t, z, \varepsilon)$ be the solution of the T-periodic $\mathcal{C}^{k+1}$ differential system (8) such that $x(0, z, \varepsilon)=z$. Then the equality

$$
x(t, z, \varepsilon)=x(t, z, 0)+\sum_{i=1}^{k} \varepsilon^{i} \frac{y_{i}(t, z)}{i !}+\mathcal{O}\left(\varepsilon^{k+1}\right)
$$

holds for $(t, z) \in \mathbb{S}^{1} \times \mathcal{D}$.

Proof. The solution $x(t, z, \varepsilon)$ can be written as

$$
\begin{aligned}
& x(t, z, \varepsilon)=z+\sum_{i=0}^{k} \varepsilon^{i} \int_{0}^{t} F_{i}(s, x(s, z, \varepsilon)) d s+\mathcal{O}\left(\varepsilon^{k+1}\right), \quad \text { and } \\
& x(t, z, 0)=z+\int_{0}^{t} F_{0}(s, x(s, z, 0)) d s .
\end{aligned}
$$

Moreover the result about differentiable dependence on parameters implies that $\varepsilon \mapsto x(t, z, \varepsilon)$ is a $\mathcal{C}^{k+1}$ map. So, for $i=0,1, \ldots, k-1$, we compute the Taylor expansion of $F_{i}(t, x(t, z, \varepsilon))$ around $\varepsilon=0$ as

$$
F_{i}(t, x(t, z, \varepsilon))=F_{i}(t, x(t, z, 0))+\left.\sum_{l=1}^{k-i} \frac{\varepsilon^{l}}{l !}\left(\frac{\partial^{l}}{\partial \varepsilon^{l}} F_{i}(t, x(t, z, \varepsilon))\right)\right|_{\varepsilon=0}+\mathcal{O}\left(\varepsilon^{k-i+1}\right) .
$$

Using the Faá di Bruno's formula to compute the $l$-derivatives of $F_{i}(t, x(t, z, \varepsilon))$ in the variable $\varepsilon$ we get

(23)

$$
\left.\frac{\partial^{l}}{\partial \varepsilon^{l}} F_{i}(t, x(t, z, \varepsilon))\right|_{\varepsilon=0}=\sum_{S_{l}} \frac{l !}{b_{1} ! b_{2} ! 2 ! b_{2} \cdots b_{l} ! l ! b_{l}} \partial^{L} F_{i}(t, x(t, z, 0)) \bigodot_{j=1}^{l} y_{j}(t, z)^{b_{j}},
$$

where

$$
y_{j}(t, z)=\left.\left(\frac{\partial^{j}}{\partial \varepsilon^{j}} x(t, z, \varepsilon)\right)\right|_{\varepsilon=0} .
$$

Substituting (23) in (22) the Taylor expansion of $F_{i}(s, x(t, z, \varepsilon))$ becomes

$$
\begin{aligned}
F_{i}(s, x(s, z, \varepsilon))= & F_{i}(s, x(s, z, 0)) \\
& +\sum_{l=1}^{k-i} \sum_{S_{l}} \frac{\varepsilon^{l}}{b_{1} ! b_{2} ! 2 ! b^{b_{2}} \cdots b_{l} ! l ! b_{l}} \partial^{L} F_{i}(s, x(s, z, 0)) \\
& \bigodot_{j=1}^{l} y_{j}(s, z)^{b_{j}}+\mathcal{O}\left(\varepsilon^{k-i+1}\right),
\end{aligned}
$$

for $i=0,1, \ldots, k-1$. Furthermore, for $i=k$,

$$
F_{k}(s, x(s, z, \varepsilon))=F_{k}(s, x(s, z, 0))+\mathcal{O}(\varepsilon) .
$$


From (21), (25), and (26), we get the following equation:

$$
x(t, z, \varepsilon)=z+Q(t, z, \varepsilon)+\sum_{i=0}^{k} \varepsilon^{i} \int_{0}^{t} F_{i}(s, x(s, z, 0)) d s+\mathcal{O}\left(\varepsilon^{k+1}\right),
$$

where

$Q(t, z, \varepsilon)=\sum_{i=1}^{k-1} \varepsilon^{i} \sum_{l=1}^{i} \sum_{S_{l}} \int_{0}^{t} \frac{1}{b_{1} ! b_{2} ! 2 ! b_{2} \cdots b_{l} ! l ! b_{l}} \partial^{L} F_{i-l}(s, x(s, z, 0)) \bigodot_{j=1}^{l} y_{j}(s, z)^{b_{j}} d s$.

Finally, from (27)

$$
\begin{aligned}
x(t, z, \varepsilon)= & z+\int_{0}^{t} F_{0}(t, x(s, z, 0)) d s+\sum_{i=1}^{k-1} \varepsilon^{i}\left(\int_{0}^{t} F_{i}(s, x(s, z, 0))\right. \\
& \left.+\sum_{l=1}^{i} \sum_{S_{l}} \frac{1}{b_{1} ! b_{2} ! 2 ! b_{2} \cdots b_{l} ! l ! l^{b_{l}}} \partial^{L} F_{i-l}(s, x(s, z, 0)) \bigodot_{j=1}^{l} y_{j}(s, z)^{b_{j}} d s\right) \\
& +\varepsilon^{k} \int_{0}^{t} F_{k}(s, x(s, z, 0))+\varepsilon^{k+1} \int_{0}^{t} R(s, x(s, z, \varepsilon), \varepsilon) d s+\mathcal{O}\left(\varepsilon^{k+1}\right) .
\end{aligned}
$$

Now using this last expression of $x(t, z, \varepsilon)$ we conclude that functions $y_{i}(t, z)$, defined in (24) for $i=1,2, \ldots, k-1$, can be computed recurrently from the following integral equation

$$
\begin{aligned}
y_{i}(t, z)= & \left.\left(\frac{\partial^{i} x}{\partial \varepsilon^{i}}(t, z, \varepsilon)\right)\right|_{\varepsilon=0} \\
= & i ! \int_{0}^{t}\left(F_{i}(s, x(s, z, 0))+\sum_{l=1}^{i} \sum_{S_{l}} \frac{1}{b_{1} ! b_{2} ! 2 ! b_{2} \cdots b_{l} ! l ! b_{l}}\right. \\
& \left.\cdot \partial^{L} F_{i-l}(s, x(s, z, 0)) \bigodot_{j=1}^{l} y_{j}(s, z)^{b_{j}}\right) d s \\
= & \int_{0}^{t}\left(A(s) y_{i}(s, z)+B_{i}(s)\right) d s,
\end{aligned}
$$

where

$$
\begin{aligned}
A(s)= & \partial F_{0}(s, x(s, z, 0)), \\
B_{i}(s)= & i !\left(F_{i}(s, x(s, z, 0))+\sum_{S_{i}^{\prime}} \frac{1}{b_{1} ! b_{2} ! 2 ! b_{2} \cdots b_{i-1} !(i-1) ! b^{b_{i-1}}} \partial^{I^{\prime}} F_{0}(s, x(s, z, 0))\right. \\
& \bigodot_{j=1}^{i-1} y_{j}(s, z)^{b_{j}}+\sum_{l=1}^{i-1} \sum_{S_{l}} \frac{1}{b_{1} ! b_{2} ! 2 ! b_{2} \cdots b_{l} ! l ! !_{l}} \partial^{L} F_{i-l}(s, x(s, z, 0)) \\
& \left.\bigodot_{j=1}^{l} y_{j}(s, z)^{b_{j}}\right) .
\end{aligned}
$$


The integral equation (28) is equivalent to the Cauchy problem

$$
\frac{\partial}{\partial t} y_{i}(t, z)=A(t) y_{i}(t, z)+B_{i}(t), \quad \text { with } \quad y_{i}(0, z)=0,
$$

which has a unique solution given by

$$
\begin{aligned}
y_{i}(t, z)= & Y(t, z) \int_{0}^{t} Y(s, z)^{-1} B_{i}(s) d s \\
= & i ! Y(t, z) \int_{0}^{t} Y(s, z)^{-1}\left(F_{i}(s, x(s, z, 0))\right. \\
& +\sum_{S_{i}^{\prime}} \frac{1}{b_{1} ! b_{2} ! 2 !^{b_{2}} \cdots b_{i} ! i !^{b_{i}}} \partial^{I^{\prime}} F_{0}(s, x(s, z, 0)) \bigodot_{j=1}^{i-1} y_{j}(s, z)^{b_{j}} \\
& \left.+\sum_{l=1}^{i-1} \sum_{S_{l}} \frac{1}{b_{1} ! b_{2} ! 2 ! !^{b_{2}} \cdots b_{l} ! l !^{b_{l}}} \partial^{L} F_{i-l}(s, x(s, z, 0)) \bigodot_{j=1}^{l} y_{j}(s, z)^{b_{j}}\right) d s .
\end{aligned}
$$

Since

$$
x(t, z, 0)=z+\int_{0}^{t} F_{0}(t, x(s, z, 0)) d s,
$$

we obtain

$$
x(t, z, \varepsilon)=x(t, z, 0)+\sum_{i=1}^{k} \varepsilon^{i} \frac{y_{i}(t, z)}{i !}+\mathcal{O}\left(\varepsilon^{k+1}\right) .
$$

This concludes the proof of the lemma

Proof of Theorem B. Let $x(\cdot, z, \varepsilon):\left[0, t_{(z, \varepsilon)}\right) \mapsto \mathbb{R}^{n}$ denote the solution of system (8) such that $x(0, z, \varepsilon)=z$. By Theorem 8.3 of [1] there exists a neighborhood $U$ of $z$ and $\varepsilon_{1}$ sufficiently small such that $t_{(z, \varepsilon)}>T$ for all $(z, \varepsilon) \in U \times\left(-\varepsilon_{1}, \varepsilon_{1}\right)$. Let $h(z, \varepsilon): U \times\left(-\varepsilon_{1}, \varepsilon_{1}\right) \mapsto \mathbb{R}^{n}$ be the displacement function defined as

$$
h(z, \varepsilon)=x(T, z, \varepsilon)-z .
$$

Clearly $x(\cdot, \bar{z}, \bar{\varepsilon})$, for some $(\bar{z}, \bar{\varepsilon}) \in U \times\left(-\varepsilon_{1}, \varepsilon_{1}\right)$, is a $T$-periodic solution of system (8) if and only if $h(\bar{z}, \bar{\varepsilon})=0$. Studying the zeros of (29) is equivalent to study the zeros of

$$
g(z, \varepsilon)=Y(T, z)^{-1} h(z, \varepsilon)
$$

From Lemma 5 we have

$$
x(t, z, \varepsilon)=x(t, z, 0)+\sum_{i=1}^{k} \varepsilon^{i} \frac{y_{i}(t, z)}{i !}+\mathcal{O}\left(\varepsilon^{k+1}\right) .
$$


for all $(t, z) \in \mathbb{S}^{1} \times \mathcal{D}$, where $y_{i}$ is defined in (12). Hence substituting (31) into (30) it follows that

$$
g(z, \varepsilon)=\sum_{i=0}^{k} \varepsilon^{i} g_{i}(z)+\mathcal{O}\left(\varepsilon^{k+1}\right),
$$

where $g_{0}(z)=Y^{-1}(t, z)(x(t, z, 0)-z)$ and, for $i=1,2, \ldots, k$, the function $g_{i}$ is defined in (11).

From hypothesis $\left(\mathrm{H}_{b}\right)$ we know that $g_{0}(z)$ vanishes on the manifold $\mathcal{Z}$, therefore hypothesis $\left(\mathrm{H}_{a}\right)$ holds for the function (32). Moreover

$$
\begin{aligned}
\frac{\partial g_{0}}{\partial z}(z)= & Y(T, z)^{-1}\left(\frac{\partial x}{\partial z}(T, z, 0)-I d\right) \\
& =Y(T, z)^{-1}\left(Y(T, z) Y(0, z)^{-1}-I d\right) \\
& =Y(0, z)^{-1}-Y(T, z)^{-1}
\end{aligned}
$$

which from hypothesis has its lower right corner $(n-m) \times(n-m)$ matrix as being a nonsingular matrix $\Delta_{\alpha}$. Hence the result follows directly by applying Theorem A.

\section{BiRTh of A Limit CYCle IN A 3D POLYNOMial SYSTEM}

Consider the following 3D autonomous polynomial differential system

$$
\begin{aligned}
\dot{u} & =-v+\varepsilon\left(u^{3}-u^{2}-u v^{2}-\pi v^{3}\right), \\
\dot{v} & =u+\varepsilon\left(\pi u^{3}-1\right) \\
\dot{w} & =w-\varepsilon u
\end{aligned}
$$

In the next proposition, as an application of Theorem B, we provide sufficient conditions for the existence of an isolated periodic solution for the differential system (33).

Proposition 6. For $|\varepsilon|>0$ sufficiently small system (33) has an isolated periodic solution $\varphi(t, \varepsilon)=(u(t, \varepsilon), v(t, \varepsilon), w(t, \varepsilon))$, such that

$$
\begin{aligned}
u(t, \varepsilon) & =\sqrt{8 \varepsilon} \cos t+\mathcal{O}(\varepsilon), \\
v(t, \varepsilon) & =\sqrt{8 \varepsilon} \sin t+\mathcal{O}(\varepsilon), \text { and } \\
w(t, \varepsilon) & =\mathcal{O}(\varepsilon) .
\end{aligned}
$$

We emphasize that the expression (34) is not saying that the period of the solution $\varphi(t, \varepsilon)$ is $2 \pi$. That is because we cannot assure the period of the order $\varepsilon$ functions. 
Proof. Writing the differential system (33) in the cylindrical coordinates $(u, v, w)=$ $(r \cos \theta, r \sin \theta, w)$ we get

$$
\begin{aligned}
\dot{r} & =\frac{\varepsilon}{4}\left(r^{3}+r^{2}(r(\pi \sin (4 \theta)+2 \cos (2 \theta)+\cos (4 \theta))-3 \cos \theta-\cos (3 \theta))-4 \sin \theta\right), \\
\dot{\theta} & =1+\frac{\varepsilon}{4 r}\left(r^{2}(\sin \theta+\sin (3 \theta)-r \sin (4 \theta)+\pi r \cos (4 \theta)+3 \pi r)-4 \cos \theta\right), \\
\dot{w} & =w-\varepsilon r \cos \theta .
\end{aligned}
$$

Since $\dot{\theta} \neq 0$ for $|\varepsilon| \neq 0$ sufficiently small, we can take $\theta$ as the new independent variable. So

$$
\begin{aligned}
& \frac{d r}{d \theta}=\varepsilon F_{11}(\theta, z)+\varepsilon^{2} F_{21}(\theta, z)+\mathcal{O}_{1}\left(\varepsilon^{3}\right) \\
& \frac{d z}{d \theta}=z+\varepsilon F_{12}(\theta, z)+\varepsilon^{2} F_{22}(\theta, z)+\mathcal{O}_{2}\left(\varepsilon^{3}\right),
\end{aligned}
$$

where $z=(r, w) \in \mathbb{R}^{2}$ and

$$
\begin{aligned}
F_{11}(\theta, z)= & \frac{1}{4}\left(r^{3}+r^{2}(r(\pi \sin (4 \theta)+2 \cos (2 \theta)+\cos (4 \theta))-3 \cos \theta-\cos (3 \theta))\right. \\
& -4 \sin \theta), \\
F_{12}(\theta, z)= & \frac{-1}{4}\left(4 \cos \theta\left(r^{2}-z\right)+r^{2} z(\sin \theta+\sin (3 \theta)-r \sin (4 \theta)+\pi r \cos (4 \theta)\right. \\
& +3 \pi r)), \\
F_{21}(\theta, z)= & \frac{-1}{16 r}\left(-4 \sin \theta+r^{3}+r^{2}(-3 \cos \theta-\cos (3 \theta)+r(\pi \sin (4 \theta)\right. \\
& +2 \cos (2 \theta)+\cos (4 \theta))))\left(r^{2}(\sin \theta+\sin (3 \theta)-r \sin (4 \theta)+\pi r \cos (4 \theta)\right. \\
& +3 \pi r)-4 \cos \theta), \\
F_{22}(\theta, z)= & \frac{1}{16 r^{2}}\left(r^{2}(\sin \theta+\sin (3 \theta)-r \sin (4 \theta)+\pi r \cos (4 \theta)+3 \pi r)-4 \cos \theta\right) \\
& \left(4 \cos \theta\left(r^{2}-z\right)+r^{2} z(\sin \theta+\sin (3 \theta)-r \sin (4 \theta)+\pi r \cos (4 \theta)\right. \\
& +3 \pi r) .
\end{aligned}
$$

The differential system (35) is $2 \pi$-periodic in the variable $\theta$ and it is written in the standard form form $(8)$ with $F_{0}(\theta, z)=(0, z), F_{1}(\theta, z)=\left(F_{11}(\theta, z), F_{12}(\theta, z)\right)$ and $F_{2}(\theta, z)=\left(F_{21}(\theta, z), F_{22}(\theta, z)\right)$. Moreover the solution of the unperturbed differential system (9) for a initial condition $z_{0}=\left(r_{0}, w_{0}\right)$ is given by

$$
\Phi\left(\theta, z_{0}\right)=\left(r_{0}, w_{0} e^{\theta}\right) .
$$

Consider the set $\mathcal{Z} \subset \mathbb{R}^{2}$ such that $\mathcal{Z}=\{(\alpha, 0): \alpha>0\}$. Clearly for each $z_{\alpha} \in \mathcal{Z}$, the solution $\Phi\left(\theta, z_{\alpha}\right)$ is $2 \pi$-periodic, and therefore the differential system 
(35) satisfies hypothesis $\left(\mathrm{H}_{b}\right)$. Furthermore the linear differential system (10) corresponding to (35) has the following fundamental matrix solution

$$
Y(\theta, z)=\frac{\partial \Phi}{\partial z}\left(\theta, z_{0}\right)=\left(\begin{array}{cc}
1 & 0 \\
0 & e^{\theta}
\end{array}\right),
$$

which satifies $Y(0, z)=I d$. Now in order to compute the bifurcation functions (5) for the differential system (35) we first obtain the functions (12) corresponding to this system,

$$
\begin{aligned}
y_{0}(\theta, z) & =Y(\theta, z)^{-1}\left(0,\left(e^{\theta}-1\right) w\right), \\
y_{1}(\theta, z) & =Y(\theta, z)^{-1}\left(\frac{r^{2}}{48}\left(-36 \sin \theta-4 \sin (3 \theta)+6 \pi r \sin ^{2}(2 \theta)+3 r \sin (4 \theta)\right)\right. \\
& \frac{1}{48}\left(12\left(\theta r^{3}-4\right)+24 \cos \theta\left(r^{3} \sin \theta+2\right)\right), \frac{r^{2}}{2}(\cos \theta-\sin \theta) \\
& -\frac{e^{\theta} r}{48}(w((36 \pi \theta-3) r+16)+24)+\frac{e^{\theta} w}{48}\left(48 \sin \theta+r^{2}(12 \cos \theta\right. \\
& +4 \cos (3 \theta)-3 r(\pi \sin (4 \theta)+\cos (4 \theta)))), \\
y_{2}(2 \pi, z) & =Y(2 \pi, z)^{-1}\left(\frac{-\pi r(3 r+4)}{4}, \frac{e^{-2 \pi}}{40}\left(((3-2 \pi) r-6) r^{2}+10\right)\right. \\
& \left.+\frac{1}{40}\left(r^{2}((\pi(7+15 \pi)-3) r+6)-10\right)\right),
\end{aligned}
$$

and from (11)

$$
g_{i}(z)=Y(2 \pi, z) \frac{y_{i}(2 \pi, z)}{i !} \quad \text { for } \quad i=0,1,2 .
$$

So the bifurcation functions (5) corresponding to the functions (36) become

$$
f_{1}(\alpha)=\frac{\pi \alpha^{3}}{2}, \quad f_{2}(\alpha)=\pi \alpha(3 \alpha+4), \quad \text { and } \quad \mathcal{F}^{2}(\alpha, \varepsilon)=\varepsilon f_{1}(\alpha)+\varepsilon^{2} f_{2}(\alpha) .
$$

Now we must check that the function (37) satisfies the hypotheses for applying Theorem B. So $\operatorname{det}\left(\Delta_{\alpha}\right)=\left|D_{w} \pi^{\perp} g_{0}\left(z_{\alpha}\right)\right|=1-e^{-2 \pi} \neq 0$, and for $a_{\varepsilon}=\sqrt{9 \varepsilon^{2}+8 \varepsilon}+3 \varepsilon$ we have that

$$
\mathcal{F}^{2}\left(a_{\varepsilon}, \varepsilon\right)=0 \quad \text { and } \quad\left|\partial_{\alpha} \mathcal{F}^{2}\left(a_{\varepsilon}, \varepsilon\right)\right| \geq \varepsilon^{2}(8-|9 \varepsilon+3 \sqrt{\varepsilon(8+9 \varepsilon)}|) .
$$

Thus it is easy to find $P_{0}>0$ satisfying $\left|\partial_{\alpha} \mathcal{F}^{2}\left(a_{\varepsilon}, \varepsilon\right)\right| \geq \varepsilon^{2} P_{0}$. Hence, in terms of Theorem $\mathrm{B}$, we have $r=1, k=2, l=2$, and $(k+r+1) / 2=2=l$. So we can apply Theorem $\mathrm{B}$ in order to prove the existence of an isolated periodic solution $(r(\theta, \varepsilon), z(\theta, \varepsilon))$ of the differential system (35) such that

$$
r(0, \varepsilon)=\sqrt{9 \varepsilon^{2}+8 \varepsilon}+3 \varepsilon+\mathcal{O}(\varepsilon)=\sqrt{8 \varepsilon}+\mathcal{O}(\varepsilon) \quad \text { and } \quad w(0, \varepsilon)=\mathcal{O}(\varepsilon) .
$$


Since $\theta(t)=t+\mathcal{O}(\varepsilon)$, this proofs ends by going back through the cylindrical coordinate change of variables.

\section{Averaged functions with a continuum of zeros}

One of the main difficulties in applying the averaging method for finding periodic solutions is to compute the zeros of the averaged function associated to the differential system. In this section we are going to show how Theorems A and B can be combined in order to deal with this problem. To be precise, consider the $T$-periodic differential system $x^{\prime}=F(t, x, \varepsilon)$ as defined in (8), with $F_{0}=0$. Note that $Y(t, z)=I d$ for every $t \in \mathbb{S}^{1}$ and $z \in D$.

As shown in the proof of Theorem $\mathrm{B}, x(t, z, \varepsilon)$ is a $T$-periodic solution of (8) if and only if $z$ is a zero of the displacement function $h$, defined in (29). In this case $h(z, \varepsilon)=g(z, \varepsilon)$, which reads

$$
h(z, \varepsilon)=x(T, z, \varepsilon)-z=\sum_{i=1}^{k} \varepsilon^{i} g_{i}(z)+O\left(\varepsilon^{k+1}\right),
$$

where the averaged functions $g_{i}(z)$, for $i=1,2, \ldots, k$, are defined in (11). In order to apply Theorem B we first compute

$$
\mathcal{F}^{k}(\alpha, \varepsilon)=\sum_{i=1}^{k} \varepsilon^{i} g_{i}(\alpha)
$$

as defined in (6), and then we try to find $a_{\varepsilon} \in V$ such that $\mathcal{F}^{k}\left(a_{\varepsilon}, \varepsilon\right)=0$. After that, if all the hypotheses of Theorem B are fulfilled we obtain, from its proof, the existence of a branch of zeros $z(\alpha)$ of the displacement function (38).

This task can be very complicate because there is no general method to find $a_{\varepsilon}$. Although if there exist $r \in\{1, \ldots, k\}$, an open subset $\widetilde{V} \subset D$, and a smooth function $\widetilde{\beta}: \operatorname{Cl}(\widetilde{V}) \rightarrow D$ such that $g_{1}=\ldots=g_{r-1}=0, g_{r} \neq 0$, and $g_{r}(\widetilde{\alpha}, \widetilde{\beta}(\widetilde{\alpha}))=$ 0 for all $\widetilde{\alpha} \subset \widetilde{V}$ then Theorem A may be used to reduce the dimension of system (39), helping then to find the solution $a_{\varepsilon}$. This strategy is a general method which generalizes the results obtained in [7]. This procedure will be illustrated in the next subsection.

6.1. Maxwell-Bloch system. In nonlinear optics, the Maxwell-Bloch equations are used to describe laser systems. For instance, in [2], these equations were obtained by coupling the Maxwell equations with the Bloch equation (a linear Schrödinger like equation which describes the evolution of atoms resonantly coupled to the laser field). Recently in [13], it was identified weak foci and centers in the Maxwell-Bloch system, which can be written as

$$
\begin{aligned}
& \dot{u}=-a u+v, \\
& \dot{v}=-b v+u w,
\end{aligned}
$$




$$
\dot{w}=-c(w-\delta)-4 u v
$$

For $c=0$ the differential system (40) has a singular line $\{(u, v, w) \mid u=0, v=0\}$; for $c \neq 0$ and $a c(\delta-a b) \leq 0$ the differential system (40) has one equilibrium $\mathbf{p}_{0}=(0,0, \delta)$; and for $c \neq 0$ and $a c(\delta-a b)>0$ the differential system (40) has three equilibria $\mathbf{p}_{ \pm}=\left( \pm u^{*}, \pm v^{*}, w^{*}\right)$ and $\mathbf{p}_{0}$ where

$$
u^{*}=\sqrt{\frac{c(\delta-a b)}{4 a}}, v^{*}=a \sqrt{\frac{c(\delta-a b)}{4 a}}, w^{*}=a b .
$$

Using the above strategy we shall prove the following result:

Proposition 7. Let $\omega \in(0, \infty),(a, b, c)=\left(a_{0}-b_{1} \varepsilon+a_{2} \varepsilon^{2},-a_{0}+b_{1} \varepsilon+b_{2} \varepsilon^{2}, c_{1} \varepsilon+\right.$ $\left.c_{2} \varepsilon^{2}\right)$ and $\delta=-a_{0}^{2}-\omega^{2}$ with $a_{0}\left(a_{2}+b_{2}\right)>0, c_{1} \neq 0$ and $\varepsilon$ a small parameter. Then for $|\varepsilon| \neq 0$ sufficiently small the Maxwell-Bloch differential system (40) has an isolated periodic solution $\varphi(t, \varepsilon)=(u(t, \varepsilon), v(t, \varepsilon), w(t, \varepsilon))$ such that

$$
\begin{aligned}
& u(t, \varepsilon)=\varepsilon \omega \sqrt{\frac{2\left(a_{2}+b_{2}\right)}{a_{0}}} \sin t+\mathcal{O}\left(\varepsilon^{2}\right), \\
& v(t, \varepsilon)=\varepsilon \omega \sqrt{\frac{2\left(a_{2}+b_{2}\right)}{a_{0}}}\left(a_{0} \sin t+\omega \cos t\right)+\mathcal{O}\left(\varepsilon^{2}\right), \text { and } \\
& w(t, \varepsilon)=\delta-\varepsilon \frac{4 \omega^{2}\left(a_{2}+b_{2}\right)}{c_{1}}+\mathcal{O}\left(\varepsilon^{2}\right) .
\end{aligned}
$$

We emphasize again that the expression (41) does not imply that the period of the solution $\varphi(t, \varepsilon)$ is $2 \pi$. That is because we cannot assure the period of the order $\varepsilon^{2}$ functions.

Proof. Applying the change of variables $(u, v, w)=\left(\varepsilon V, \varepsilon\left(a_{0} V+\omega U\right), \delta+\varepsilon W\right)$, the differential system (40) reads

$$
\begin{aligned}
\dot{U} & =-\omega V+\frac{\varepsilon}{\omega}\left(V W-2 a_{0} b_{1} V-b_{1} \omega U\right)+\varepsilon^{2}\left(\frac{a_{0}\left(a_{2}-b_{2}\right) V}{\omega}-b_{2} U\right), \\
\dot{V} & =\omega U+\varepsilon b_{1} V-\varepsilon^{2} a_{2} V \\
\dot{W} & =\varepsilon\left(-c_{1} W-4 V\left(a_{0} V+\omega U\right)\right)-\varepsilon^{2} c_{2} .
\end{aligned}
$$

In order to apply the strategy described above we must write the differential system (42) in the standard form (8). To this end we proceed as usual: first we consider the cylindrical change of variables $(U, V, W)=(r \cos \theta, r \sin \theta, w)$, where $r>0$; after checking that $\dot{\theta}=\omega+\mathcal{O}(\varepsilon) \neq 0$, for $|\varepsilon| \neq 0$ sufficiently small, we take $\theta$ as the new independent variable. Therefore the differential system (42) becomes equivalent to the non-autonomous differential system

$$
\frac{d z}{d \theta}=\left(\frac{\dot{r}}{\dot{\theta}}, \frac{\dot{w}}{\dot{\theta}}\right)=\varepsilon F_{1}(\theta, z)+\varepsilon^{2} F_{2}(\theta, z)+\mathcal{O}\left(\varepsilon^{3}\right),
$$


where $z=(r, w) \in \mathbb{R}^{+} \times \mathbb{R}$ and $\theta \in \mathbb{S}^{1}$. Moreover

(44)

$$
\begin{aligned}
F_{1}(\theta, z)= & \left(\frac{r}{2 \omega^{2}}\left(\left(w-2 a_{0} b_{1}\right) \sin (2 \theta)-2 b_{1} \omega \cos (2 \theta)\right),\right. \\
& \left.-\frac{\left(c_{1} w+4 r^{2} \sin \theta\left(\omega \cos \theta+a_{0} \sin \theta\right)\right)}{\omega}\right), \\
F_{2}(\theta, z)= & \left(\frac { 1 } { 2 \omega ^ { 4 } } ( 2 b _ { 1 } \omega \operatorname { c o s } \theta + ( 2 a _ { 0 } b _ { 1 } - w ) \operatorname { s i n } \theta ) \left(2 b_{1} \omega \cos (2 \theta)+\left(2 a_{0} b_{1}\right.\right.\right. \\
& -w) \sin (2 \theta))+r \omega^{2}\left(\left(a_{2}-b_{2}\right)\left(\omega \cos (2 \theta)+a_{0} \sin (2 \theta)\right)-\left(a_{2}+b_{2}\right)\right), \\
& \left.\frac{\left(2 b_{1} \omega \cos \theta+\left(2 a_{0} b_{1}-w\right) \sin \theta\right)}{\omega^{2}}\left(c_{1} w+4 r^{2} \sin \theta\left(\omega \cos \theta+a_{0} \sin \theta\right)\right)\right) .
\end{aligned}
$$

Now the prime denotes the derivative with respect to the variable $\theta$.

For the differential system (43) we have that $F_{0}(\theta, z)=0$. Then $x(\theta, z, 0)=$ $(r, w)$ is the solution to the unperturbed system and $Y(t, z)=I d$ is its corresponding fundamental matrix. In this case the averaged functions reads

$$
\begin{aligned}
g_{1}(z)= & \left(0,-\frac{2 \pi\left(2 a_{0} r^{2}+c_{1} w\right)}{\omega}\right) \\
g_{2}(z)= & \left(\frac{\pi r\left(3 a_{0} r^{2}+c_{1} w-2\left(a_{2}+b_{2}\right) \omega^{2}\right)}{2 \omega^{3}}, \frac{\pi}{\omega^{3}}\left(\left(2 a_{0} b_{1}-w\right)\left(6 a_{0} r^{2}+c_{1} w\right)\right.\right. \\
& \left.\left.+2 c_{1} \pi\left(2 a_{0} r^{2}+c_{1} w\right) \omega+2\left(\left(2 b_{1}+c_{1}\right) r^{2}-c_{2} w\right) \omega^{2}\right)\right) .
\end{aligned}
$$

From here instead of following the steps of Theorem B we are going to use Theorem A to find directly a branch of zeros of the displacement function (38). To do this we define the function $\widetilde{g}(z, \varepsilon)=h(z, \varepsilon) / \varepsilon$, where now $\widetilde{g}(z, \varepsilon)=\widetilde{g}_{0}(z)+$ $\varepsilon \widetilde{g}_{1}(z)+\mathcal{O}\left(\varepsilon^{2}\right)$ with $\widetilde{g}_{0}(z)=g_{1}(z)$ and $\widetilde{g}_{1}(z)=g_{2}(z)$. Note that the averaged function $\widetilde{g}_{0}(z)=g_{1}(z)$ vanishes on the manifold

$$
\widetilde{\mathcal{Z}}=\left\{z_{\alpha}=\left(\alpha,-\frac{2 a_{0} \alpha^{2}}{c_{1}}\right): \alpha>0\right\} .
$$

Furthermore, $\Delta_{\alpha}=-\left(2 \pi c_{1}\right) / \omega$ is the lower right corner of the Jacobian matrix $D \widetilde{g}_{0}\left(z_{\alpha}\right)$ for all $z_{\alpha} \in \widetilde{\mathcal{Z}}$. Computing then the bifurcation function (5) corresponding to $\widetilde{g}(z, \varepsilon)$ we get

$$
\widetilde{f}_{1}(\alpha)=\frac{\pi \alpha\left(a_{0} \alpha^{2}-2\left(a_{2}+b_{2}\right) \omega^{2}\right)}{2 \omega^{3}}
$$


and $\widetilde{\mathcal{F}}^{1}(\alpha, \varepsilon)=\varepsilon \widetilde{f}_{1}(\alpha)$. Solving the equation $\widetilde{\mathcal{F}}^{1}(\alpha, \varepsilon)=0$ we find

$$
a_{\varepsilon}=\alpha_{0}=\omega \sqrt{\frac{2\left(a_{2}+b_{2}\right)}{a_{0}}} .
$$

Moreover, $\left|\partial_{\alpha} \widetilde{\mathcal{F}}^{1}\left(\alpha_{0}, \varepsilon\right)\right|=2 \varepsilon \pi\left(a_{2}+b_{2}\right) / \omega$ so it is clear that hypotheses $(i i i)$ and (iv) of Theorem A are fulfilled with $l=1, r=1$ and $k=1$. Thus, for $|\varepsilon| \neq 0$ sufficiently small, it follows that there exists

$$
z(\varepsilon)=\left(\omega \sqrt{\frac{2\left(a_{2}+b_{2}\right)}{a_{0}}},-\frac{4 \omega^{2}\left(a_{2}+b_{2}\right)}{c_{1}}\right)+\mathcal{O}(\varepsilon)
$$

such that $\widetilde{g}(z(\varepsilon), \varepsilon)=h(z(\varepsilon), \varepsilon) / \varepsilon=0$ for every $|\varepsilon| \neq 0$ sufficiently small. Therefore we conclude that there exists a $2 \pi$-periodic solution periodic $(r(\theta, \varepsilon), w(\theta, \varepsilon))$ of the non-autonomous differential system (43) satisfying $(r(\theta, 0), w(\theta, 0))=z(0)$. Since $\theta(t)=\omega t+\mathcal{O}(\varepsilon)$, this proofs ends by going back through the cylindrical coordinate change of variables and then doing $(u, v, z)=\varepsilon\left(V, a_{0} V+\omega U, W\right)$.

6.2. Stability. We have seen that the averaged functions (45) up to order 2 were sufficient for detecting the existence of a periodic solution of the differential system (40). Now we show that the higher order averaged functions may play an important role for studying the stability of the periodic solution $\varphi(t, \varepsilon)$ provided by Theorem B.

Clearly the stability of the periodic solution $\varphi(t, \varepsilon)$ can be derived from the eigenvalues of the Jacobian matrix of the displacement function $D_{z} h(z(\varepsilon), \varepsilon)$ evaluated at $z(\varepsilon)=\varphi(0, \varepsilon)$. From equation (46) we can write $z(\varepsilon)=z_{0}+\mathcal{O}\left(\varepsilon^{2}\right)$. Moreover, since in this case $Y(t, z)=I d$ then $D_{z} h(z(\varepsilon), \varepsilon)=\varepsilon D g_{1}\left(z_{0}\right)+\mathcal{O}(\varepsilon)$, where

$$
D g_{1}\left(z_{0}\right)=\left(\begin{array}{cc}
0 & 0 \\
-8 \pi \sqrt{2 a_{0}\left(a_{2}+b_{2}\right)} & -\frac{2 \pi c_{1}}{\omega}
\end{array}\right) .
$$

So a first approximation of the eigenvalues $\lambda_{ \pm}$of the Jacobian matrix $D_{z} h(z(\varepsilon), \varepsilon)$ is given by

$$
\lambda_{+}=\mathcal{O}\left(\varepsilon^{2}\right), \quad \lambda_{-}=-\varepsilon \frac{2 \pi c_{1}}{\omega}+\mathcal{O}\left(\varepsilon^{2}\right) .
$$

Clearly the stability of the periodic solution $\varphi(t, \varepsilon)$ cannot be completely described by these expressions. Now we show how the higher order bifurcation functions and averaging functions can be used to better analyses the stability of the periodic solution.

We recall that, after some changes of coordinates, the differential system (40) can be transformed into the standard form (43). Expanding it in power series of 
$\varepsilon$ up to order 3, the differential system (43) becomes

$$
\frac{d z}{d \theta}=\varepsilon F_{1}(\theta, z)+\varepsilon^{2} F_{2}(\theta, z)+\varepsilon^{3} F_{3}(\theta, z)+\mathcal{O}\left(\varepsilon^{4}\right),
$$

where $F_{1}$ and $F_{2}$ are given in (44) and

$$
\begin{aligned}
F_{3}(\theta, z)= & \frac{\pi r}{4 \omega^{5}}\left(-3\left(a_{0} b_{1}-w\right)\left(5 a_{0} r^{2}+c_{1} w\right)-2 c_{1} \pi\left(2 a_{0} r^{2}+c_{1} w\right) \omega+\left(4 a _ { 0 } b _ { 1 } \left(a_{2}\right.\right.\right. \\
& \left.\left.\left.+b_{2}\right)-3\left(2 b_{1}+c_{1}\right) r^{2}-2\left(a_{2}+b_{2}-c_{2}\right) w\right) \omega^{2}\right) \\
& \frac{\pi}{12 \omega^{5}}\left(12 \pi \omega\left(a_{0}^{2}\left(6 r^{4}-16 b_{1} c_{1} r^{2}\right)+2 a_{0} c_{1} w\left(7 r^{2}-2 b_{1} c_{1}\right)+3 c_{1}^{2} w^{2}\right)\right. \\
& -2 \omega^{2}\left(w\left(6 a_{0}\left(a_{2} c_{1}-2 b_{1} c_{2}-b_{2} c_{1}\right)+6 b_{1}^{2} c_{1}-9 r^{2}\left(4 b_{1}+3 c_{1}\right)+8 \pi^{2} c_{1}^{3}\right)\right. \\
& \left.+a_{0} r^{2}\left(36 a_{0}\left(a_{2}-b_{2}\right)+108 b_{1}^{2}+36 b_{1} c_{1}+2\left(8 \pi^{2}-3\right) c_{1}^{2}-45 r^{2}\right)+6 c_{2} w^{2}\right) \\
& +24 \pi \omega^{3}\left(r^{2}\left(2 a_{0}\left(a_{2}+b_{2}+c_{2}\right)-c_{1}\left(2 b_{1}+c_{1}\right)\right)+2 c_{1} c_{2} w\right) \\
& \left.-9\left(w-2 a_{0} b_{1}\right)^{2}\left(10 a_{0} r^{2}+c_{1} w\right)+24 r^{2} \omega^{4}\left(c_{2}-2 a_{2}\right)\right) .
\end{aligned}
$$

From (12) and (5) we compute the third averaged function and the second bifurcation function, respectively, as

$$
\begin{aligned}
g_{3}(z)= & \left(\frac { \pi r } { 4 \omega ^ { 5 } } \left(\omega^{2}\left(4 a_{0} b_{1}\left(a_{2}+b_{2}\right)-2 z\left(a_{2}+b_{2}-c_{2}\right)-3 r^{2}\left(2 b_{1}+c_{1}\right)\right)\right.\right. \\
& \left.-3\left(2 a_{0} b_{1}-z\right)\left(5 a_{0} r^{2}+c_{1} z\right)-2 \pi c_{1} \omega\left(2 a_{0} r^{2}+c_{1} z\right)\right), \\
& \frac{\pi}{12 \omega^{5}}\left(12 \pi \omega\left(a_{0}^{2}\left(6 r^{4}-16 b_{1} c_{1} r^{2}\right)+2 a_{0} c_{1} z\left(7 r^{2}-2 b_{1} c_{1}\right)+3 c_{1}^{2} z^{2}\right)\right. \\
& -2 \omega^{2}\left(z\left(6 a_{0}\left(a_{2} c_{1}-2 b_{1} c_{2}-b_{2} c_{1}\right)+6 b_{1}^{2} c_{1}-9 r^{2}\left(4 b_{1}+3 c_{1}\right)+8 \pi^{2} c_{1}^{3}\right)\right. \\
& +a_{0} r^{2}\left(36 a_{0}\left(a_{2}-b_{2}\right)+108 b_{1}^{2}+36 b_{1} c_{1}+2\left(8 \pi^{2}-3\right) c_{1}^{2}-45 r^{2}\right) \\
& \left.+6 c_{2} z^{2}\right)+24 \pi \omega^{3}\left(r^{2}\left(2 a_{0}\left(a_{2}+b_{2}+c_{2}\right)-c_{1}\left(2 b_{1}+c_{1}\right)\right)+2 c_{1} c_{2} z\right) \\
& \left.\left.-9\left(z-2 a_{0} b_{1}\right)^{2}\left(10 a_{0} r^{2}+c_{1} z\right)+24 r^{2} \omega^{4}\left(c_{2}-2 a_{2}\right)\right)\right)
\end{aligned}
$$

and

$$
\widetilde{f}_{2}(\alpha)=-\frac{\pi r\left(10 a_{0}^{2} r^{2}\left(b_{1} c_{1}+r^{2}\right)+\omega^{2}\left(c_{1} r^{2}\left(2 b_{1}+c_{1}\right)-4 a_{0}\left(a_{2}+b_{2}\right)\left(b_{1} c_{1}+r^{2}\right)\right)\right)}{4 c_{1} \omega^{5}} .
$$

So $\widetilde{\mathcal{F}}^{2}(\alpha, \varepsilon)=\varepsilon \widetilde{f}_{1}(\alpha)+\varepsilon^{2} \widetilde{f}_{2}(\alpha)$. As shown in the previous subsection $a_{\varepsilon}=\alpha_{0}$ is a simple root of the function $\tilde{f}_{1}(\alpha)$. Using the Implicit Function Theorem we 
find a branch of zeros of the equation $\mathcal{F}^{2}(\alpha, \varepsilon)=0$ having the form $\alpha=\bar{a}_{\varepsilon}=$ $\alpha_{0}+\varepsilon \alpha_{1}+\mathcal{O}\left(\varepsilon^{2}\right)$, where

$$
\alpha_{1}=\sqrt{\frac{a_{2}+b_{2}}{2 a_{0}}}\left(\frac{8 a_{0}^{2} b_{1} c_{1}+\omega^{2}\left(16 a_{0}\left(a_{2}+b_{2}\right)+c_{1}\left(2 b_{1}+c_{1}\right)\right)}{2\left|a_{0}\right| c_{1} \omega}\right) .
$$

Note that $\bar{a}_{\varepsilon}$ satisfies the hypotheses (iii) and (iv) of Theorem A for $r=1, l=1$ and $k=2$. Using the relation $\left|\pi z(\varepsilon)-\pi z_{\bar{a}_{\varepsilon}}\right|=\left|\alpha(\varepsilon)-\bar{a}_{\varepsilon}\right|=\mathcal{O}\left(\varepsilon^{2}\right)$, provided by Theorem A, we write $\alpha(\varepsilon)=\alpha_{0}+\varepsilon \alpha_{1}+\mathcal{O}\left(\varepsilon^{2}\right)$. From Claim 1 of the proof of Theorem A we get

$$
\begin{aligned}
\bar{\beta}(\alpha(\varepsilon), \varepsilon) & =\beta(\alpha(\varepsilon))+\varepsilon \gamma_{1}(\alpha(\varepsilon))+\mathcal{O}\left(\varepsilon^{2}\right) \\
& =\beta\left(\alpha_{0}+\varepsilon \alpha_{1}+\mathcal{O}\left(\varepsilon^{2}\right)\right)+\varepsilon \gamma_{1}\left(\alpha_{0}+\varepsilon \alpha_{1}+\mathcal{O}\left(\varepsilon^{2}\right)\right)+\mathcal{O}\left(\varepsilon^{2}\right) .
\end{aligned}
$$

Expanding $\bar{\beta}(\alpha(\varepsilon), \varepsilon)$ in powers series of $\varepsilon$ we have $\bar{\beta}(\alpha(\varepsilon), \varepsilon)=\beta_{0}+\varepsilon \beta_{1}+\mathcal{O}\left(\varepsilon^{2}\right)$ where

$$
\begin{aligned}
& \beta_{0}=\frac{\left(a_{2}+b_{2}\right) \omega^{2}}{c_{1}}, \\
& \beta_{1}=\frac{4\left(a_{2}+b_{2}\right)\left(6 a_{0}^{2} b_{1} c_{1}+\left(16 a_{0}\left(a_{2}+b_{2}\right)+c_{1}\left(2 b_{1}+c_{1}\right)\right) \omega^{2}\right)}{a_{0} c_{1}^{2}} .
\end{aligned}
$$

Finally we obtain $z(\varepsilon)=(\alpha(\varepsilon), \bar{\beta}(\alpha(\varepsilon), \varepsilon))=z_{0}+\varepsilon z_{1}+\mathcal{O}\left(\varepsilon^{2}\right)$, with $z_{0}=\left(\alpha_{0}, \beta_{0}\right)$ and $z_{1}=\left(\alpha_{1}, \beta_{1}\right)$. Then we compute the Jacobian matrix of the displacement function $(29)$ evaluated at $z(\varepsilon)$ as

$$
\begin{aligned}
D_{z} h(z(\varepsilon), \varepsilon) & =\varepsilon D_{z} g_{1}(z(\varepsilon))+\varepsilon^{2} D_{z} g_{2}(z(\varepsilon))+\mathcal{O}\left(\varepsilon^{3}\right) \\
& =\varepsilon D_{z} g_{1}\left(z_{0}+\varepsilon z_{1}+\mathcal{O}\left(\varepsilon^{2}\right)\right)+\varepsilon^{2} D_{z} g_{2}\left(z_{0}+\varepsilon z_{1}+\mathcal{O}\left(\varepsilon^{2}\right)\right)+\mathcal{O}\left(\varepsilon^{3}\right) \\
& =\varepsilon D_{z} g_{1}\left(z_{0}\right)+\varepsilon^{2}\left(D_{z}^{2} g_{1}\left(z_{0}\right) z_{1}+D_{z} g_{2}\left(z_{0}\right)\right)+\mathcal{O}\left(\varepsilon^{3}\right)
\end{aligned}
$$

Let $D_{z} g_{1}\left(z_{0}\right)=\left(p_{i j}\right)_{2 \times 2}$ and $D_{z} g_{2}\left(z_{0}\right)=\left(q_{i j}\right)_{2 \times 2}$ then expanding $D_{z} h(z(\varepsilon), \varepsilon)$ in Taylor series around $\varepsilon=0$ we have $D_{z} h(z(\varepsilon), \varepsilon)=\varepsilon A_{1}+\varepsilon^{2} A_{2}+\mathcal{O}\left(\varepsilon^{3}\right)$ with $A_{1}=D_{z} g_{1}\left(z_{0}\right)$ and $A_{2}=\left(D_{z} p_{i j}\left(z_{0}\right) . z_{1}+q_{i j}\left(z_{0}\right)\right)_{2 \times 2}$. Therefore we may improve the approximation (47) of the eigenvalues $\lambda_{ \pm}$of $D_{z} h(z(\varepsilon), \varepsilon)$ as

$$
\begin{aligned}
& \lambda_{+}=\varepsilon^{2} \frac{2 \pi\left(a_{2}+b_{2}\right)}{\omega}+\mathcal{O}\left(\varepsilon^{3}\right), \\
& \lambda_{-}=-\varepsilon \frac{2 c_{1} \pi}{\omega}+\varepsilon^{2} \frac{2 \pi\left(a_{0} b_{1} c_{1}+\omega\left(c_{1}^{2} \pi-c_{2} \omega\right)\right)}{\omega^{3}}+\mathcal{O}\left(\varepsilon^{3}\right) .
\end{aligned}
$$

Hence we can deduce the following statements about the stability of the periodic solution $\varphi(t, \varepsilon)=x(t, z(\varepsilon), \varepsilon)$. Recall that from, hypotheses of Proposition 7, $a_{0}\left(a_{2}+b_{2}\right)>0$. So:

(a) If $\varepsilon c_{1}<0$ the solution $\varphi(t, \varepsilon)$ has at least one unstable direction, 
(b) If $a_{2}+b_{2}>0$ and $a_{0}>0$ then the solution $\varphi(t, \varepsilon)$ has at least one unstable direction,

(c) If $a_{2}+b_{2}<0, \varepsilon c_{1}>0$ and $a_{0}<0$ then the solution $\varphi(t, \varepsilon)$ is asymptotically stable.

The following figures illustrate the behavior of the Maxwell-Block system (40) satisfying the hypotheses of Proposition 7 with $a_{0}=-1, a_{2}=-2, b_{1}=1$, $b_{2}=-2, c_{1}=2, c_{2}=1, \omega=1$ and $\varepsilon=1 / 25$.

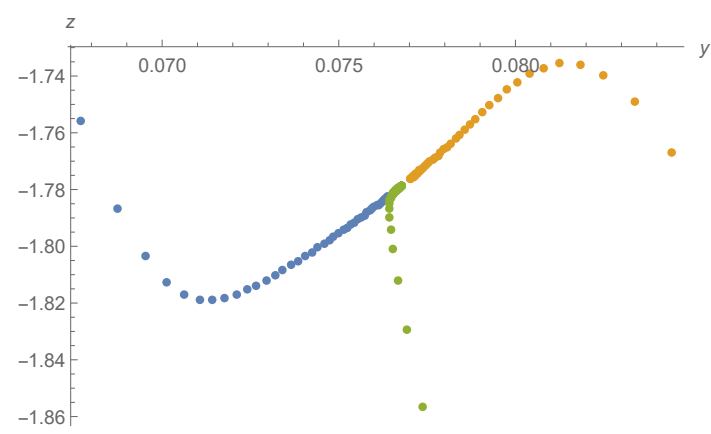

(A)

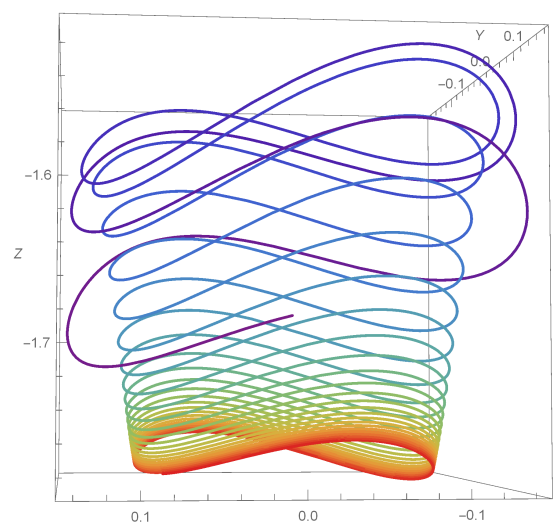

(B)

Figure 1. (A) Transversal section with $u=0$ and $v>0$. (B) Solution starting at $\left(0, \varepsilon \omega^{2}\left(2\left(a_{2}+b_{2}\right) / a_{0}\right)^{1 / 2}, \delta-4 \varepsilon \omega^{2}\left(a_{2}+b_{2}\right) / c_{1}\right)$ being attracted by the limit cycle (41).

\section{APPENDIX: BIFURCATION FUnCTIONS UP TO ORDER 5.}

In this appendix we develop the recurrences given by Theorems A and B to compute explicitly the expressions of the bifurcation functions and the averaged functions up to order 5. As far as we know we are the first to provide these expressions.

From the recurrences (5) and (7), we explicitly develop the expressions of the bifurcation functions $f_{i}: V \rightarrow \mathbb{R}^{m}$, for $i=1,2, . ., 5$, as stated in Theorem A. Recall that $\Gamma_{\alpha}=\left(\partial \pi g_{0} / \partial b\right)\left(z_{\alpha}\right)$. So

$f_{1}(\alpha)=\Gamma_{\alpha} \gamma_{1}(\alpha)+\pi g_{1}\left(z_{\alpha}\right)$,

$\gamma_{1}(\alpha)=-\Delta_{\alpha}^{-1} \pi^{\perp} g_{1}\left(z_{\alpha}\right)$,

$f_{2}(\alpha)=\frac{1}{2} \Gamma_{\alpha} \gamma_{2}(\alpha)+\frac{1}{2} \frac{\partial^{2} \pi g_{0}}{\partial b^{2}}\left(z_{\alpha}\right) \gamma_{1}(\alpha)^{2}+\frac{\partial \pi g_{1}}{\partial b}\left(z_{\alpha}\right) \gamma_{1}(\alpha)+\pi g_{2}\left(z_{\alpha}\right)$, 


$$
\begin{aligned}
& \gamma_{2}(\alpha)=-\Delta_{\alpha}^{-1}\left(\frac{\partial^{2} \pi^{\perp} g_{0}}{\partial b^{2}}\left(z_{\alpha}\right) \gamma_{1}(\alpha)^{2}+2 \frac{\partial \pi^{\perp} g_{1}}{\partial b}\left(z_{\alpha}\right) \gamma_{1}(\alpha)+2 \pi^{\perp} g_{2}(\alpha)\right), \\
& f_{3}(\alpha)=\frac{1}{6} \Gamma_{\alpha} \gamma_{3}(\alpha)+\frac{1}{6} \frac{\partial^{3} \pi g_{0}}{\partial b^{3}}\left(z_{\alpha}\right) \gamma_{1}(\alpha)^{3}+\frac{1}{2} \frac{\partial^{2} \pi g_{0}}{\partial b^{2}}\left(z_{\alpha}\right) \gamma_{1}(\alpha) \odot \gamma_{2}(\alpha) \\
& +\frac{1}{2} \frac{\partial^{2} \pi g_{1}}{\partial b^{2}}\left(z_{\alpha}\right) \gamma_{1}(\alpha)^{2}+\frac{1}{2} \frac{\partial \pi g_{1}}{\partial b}\left(z_{\alpha}\right) \gamma_{2}(\alpha)+\frac{\partial \pi g_{2}}{\partial b}\left(z_{\alpha}\right) \gamma_{1}(\alpha) \\
& +\pi g_{3}\left(z_{\alpha}\right) \\
& \gamma_{3}(\alpha)=-\Delta_{\alpha}^{-1}\left(\frac{\partial^{3} \pi^{\perp} g_{0}}{\partial b^{3}}\left(z_{\alpha}\right) \gamma_{1}(\alpha)^{3}+3 \frac{\partial^{2} \pi^{\perp} g_{0}}{\partial b^{2}}\left(z_{\alpha}\right) \gamma_{1}(\alpha) \odot \gamma_{2}(\alpha)\right. \\
& +3 \frac{\partial^{2} \pi^{\perp} g_{1}}{\partial b^{2}}\left(z_{\alpha}\right) \gamma_{1}(\alpha)^{2}+2 \frac{\partial \pi^{\perp} g_{1}}{\partial b}\left(z_{\alpha}\right) \gamma_{2}(\alpha)+6 \frac{\partial \pi^{\perp} g_{2}}{\partial b}\left(z_{\alpha}\right) \gamma_{1}(\alpha) \\
& \left.+6 \pi^{\perp} g_{3}(\alpha)\right) \\
& f_{4}(\alpha)=\frac{1}{24} \Gamma_{\alpha} \gamma_{4}(\alpha)+\frac{1}{24} \frac{\partial^{4} \pi g_{0}}{\partial b^{4}}\left(z_{\alpha}\right) \gamma_{1}(\alpha)^{4}+\frac{1}{4} \frac{\partial^{3} \pi g_{0}}{\partial b^{3}}\left(z_{\alpha}\right) \gamma_{1}(\alpha)^{2} \odot \gamma_{2}(\alpha) \\
& +\frac{1}{8} \frac{\partial^{2} \pi g_{0}}{\partial b^{2}}\left(z_{\alpha}\right) \gamma_{2}(\alpha)^{2}+\frac{1}{6} \frac{\partial^{2} \pi g_{0}}{\partial b^{2}}\left(z_{\alpha}\right) \gamma_{1}(\alpha) \odot \gamma_{3}(\alpha) \\
& +\frac{1}{6} \frac{\partial^{3} \pi g_{1}}{\partial b^{3}}\left(z_{\alpha}\right) \gamma_{1}(\alpha)^{3}+\frac{1}{2} \frac{\partial^{2} \pi g_{1}}{\partial b^{2}}\left(z_{\alpha}\right) \gamma_{1}(\alpha) \odot \gamma_{2}(\alpha)+\frac{1}{6} \frac{\partial \pi g_{1}}{\partial b}\left(z_{\alpha}\right) \gamma_{3}(\alpha) \\
& +\frac{1}{2} \frac{\partial^{2} \pi g_{2}}{\partial b^{2}}\left(z_{\alpha}\right) \gamma_{1}(\alpha)^{2}+\frac{1}{2} \frac{\partial \pi g_{2}}{\partial b}\left(z_{\alpha}\right) \gamma_{2}(\alpha)+\frac{\partial \pi g_{3}}{\partial b}\left(z_{\alpha}\right) \gamma_{1}(\alpha)+\pi g_{4}\left(z_{\alpha}\right), \\
& \gamma_{4}(\alpha)=-\Delta_{\alpha}^{-1}\left(\frac{\partial^{4} \pi^{\perp} g_{0}}{\partial b^{4}}\left(z_{\alpha}\right) \gamma_{1}(\alpha)^{4}+3 \frac{\partial^{2} \pi^{\perp} g_{0}}{\partial b^{2}}\left(z_{\alpha}\right) \gamma_{2}(\alpha)^{2}+4 \frac{\partial^{2} \pi^{\perp} g_{0}}{\partial b^{2}}\left(z_{\alpha}\right) \gamma_{1}(\alpha) \odot \gamma_{3}(\alpha)\right. \\
& +6 \frac{\partial^{3} \pi^{\perp} g_{0}}{\partial b^{3}}\left(z_{\alpha}\right) \gamma_{1}(\alpha)^{2} \odot \gamma_{2}(\alpha)+4 \frac{\partial \pi^{\perp} g_{1}}{\partial b}\left(z_{\alpha}\right) \gamma_{3}(\alpha)+12 \frac{\partial^{2} \pi^{\perp} g_{1}}{\partial b^{2}}\left(z_{\alpha}\right) \gamma_{1}(\alpha) \odot \gamma_{2}(\alpha) \\
& +4 \frac{\partial^{3} \pi^{\perp} g_{1}}{\partial b^{3}}\left(z_{\alpha}\right) \gamma_{1}(\alpha)^{3}+12 \frac{\partial \pi^{\perp} g_{2}}{\partial b}\left(z_{\alpha}\right) \gamma_{2}(\alpha)+12 \frac{\partial^{2} \pi^{\perp} g_{2}}{\partial b^{2}}\left(z_{\alpha}\right) \gamma_{1}(\alpha)^{2} \\
& \left.+24 \frac{\partial \pi^{\perp} g_{3}}{\partial b}\left(z_{\alpha}\right) \gamma_{1}(\alpha)\right) \\
& f_{5}(\alpha)=\frac{1}{120} \Gamma_{\alpha} \gamma_{5}(\alpha)+\frac{1}{12} \frac{\partial^{2} \pi g_{0}}{\partial b^{2}}\left(z_{\alpha}\right) \gamma_{2}(\alpha) \odot \gamma_{1}(\alpha)+\frac{1}{24} \frac{\partial^{2} \pi g_{0}}{\partial b^{2}}\left(z_{\alpha}\right) \gamma_{1}(\alpha) \odot \gamma_{4}(\alpha) \\
& +\frac{1}{8} \frac{\partial^{3} \pi g_{0}}{\partial b^{3}}\left(z_{\alpha}\right) \gamma_{1}(\alpha) \odot \gamma_{2}(\alpha)^{2}+\frac{1}{12} \frac{\partial^{3} \pi g_{0}}{\partial b^{3}}\left(z_{\alpha}\right) \gamma_{1}(\alpha)^{2} \odot \gamma_{3}(\alpha) \\
& +\frac{1}{12} \frac{\partial^{4} \pi g_{0}}{\partial b^{4}}\left(z_{\alpha}\right) \gamma_{1}(\alpha)^{3} \odot \gamma_{2}(\alpha)+\frac{1}{120} \frac{\partial^{5} \pi g_{0}}{\partial b^{5}}\left(z_{\alpha}\right) \gamma_{1}(\alpha)^{5}
\end{aligned}
$$




$$
\begin{aligned}
+ & \frac{1}{24} \frac{\partial \pi g_{1}}{\partial b}\left(z_{\alpha}\right) \gamma_{4}(\alpha)+\frac{1}{8} \frac{\partial^{2} \pi g_{1}}{\partial b^{2}}\left(z_{\alpha}\right) \gamma_{2}(\alpha)^{2}+\frac{1}{6} \frac{\partial^{2} \pi g_{1}}{\partial b^{2}}\left(z_{\alpha}\right) \gamma_{1}(\alpha) \odot \gamma_{3}(\alpha) \\
& +\frac{1}{4} \frac{\partial^{3} \pi g_{1}}{\partial b^{3}}\left(z_{\alpha}\right) \gamma_{1}(\alpha)^{2} \odot \gamma_{2}(\alpha)+\frac{1}{24} \frac{\partial^{4} \pi g_{1}}{\partial b^{4}}\left(z_{\alpha}\right) \gamma_{1}(\alpha)^{4}+\frac{1}{6} \frac{\partial \pi g_{2}}{\partial b}\left(z_{\alpha}\right) \gamma_{3}(\alpha) \\
& +\frac{1}{2} \frac{\partial^{2} \pi g_{2}}{\partial b^{2}}\left(z_{\alpha}\right) \gamma_{1}(\alpha) \odot \gamma_{2}(\alpha)+\pi g_{4}\left(z_{\alpha}\right)+\frac{1}{6} \frac{\partial^{3} \pi g_{2}}{\partial b^{3}}\left(z_{\alpha}\right) \gamma_{1}(\alpha)^{3} \\
& +\frac{1}{2} \frac{\partial \pi g_{3}}{\partial b}\left(z_{\alpha}\right) \gamma_{2}(\alpha)+\frac{1}{2} \frac{\partial^{2} \pi g_{3}}{\partial b^{2}}\left(z_{\alpha}\right) \gamma_{1}(\alpha)^{2}+\frac{\partial \pi g_{4}}{\partial b}\left(z_{\alpha}\right) \gamma_{1}(\alpha) \\
& +\pi g_{5}\left(z_{\alpha}\right), \\
\gamma_{5}(\alpha)= & -\Delta \frac{-1}{\alpha}\left(10 \frac{\partial^{2} \pi^{\perp} g_{0}}{\partial b^{2}}\left(z_{\alpha}\right) \gamma_{2}(\alpha) \odot \gamma_{3}(\alpha)+5 \frac{\partial^{2} \pi^{\perp} g_{0}}{\partial b^{2}}\left(z_{\alpha}\right) \gamma_{1}(\alpha) \odot \gamma_{4}(\alpha)\right. \\
& +15 \frac{\partial^{3} \pi^{\perp} g_{0}}{\partial b^{3}}\left(z_{\alpha}\right) \gamma_{1}(\alpha) \odot \gamma_{2}(\alpha)^{2}+10 \frac{\partial^{3} \pi^{\perp} g_{0}}{\partial b^{3}}\left(z_{\alpha}\right) \gamma_{1}(\alpha)^{2} \odot \gamma_{3}(\alpha) \\
& +10 \frac{\partial^{4} \pi^{\perp} g_{0}}{\partial b^{4}}\left(z_{\alpha}\right) \gamma_{1}(\alpha)^{3} \odot \gamma_{2}(\alpha)+\frac{\partial^{5} \pi^{\perp} g_{0}}{\partial b^{5}}\left(z_{\alpha}\right) \gamma_{1}(\alpha)^{5} \\
& +5 \frac{\partial \pi^{\perp} g_{1}}{\partial b}\left(z_{\alpha}\right) \gamma_{4}(\alpha)+15 \frac{\partial^{2} \pi^{\perp} g_{1}}{\partial b^{2}}\left(z_{\alpha}\right) \gamma_{2}(\alpha)^{2}+20 \frac{\partial^{2} \pi^{\perp} g_{1}}{\partial b^{2}}\left(z_{\alpha}\right) \gamma_{1}(\alpha) \odot \gamma_{3}(\alpha) \\
& +30 \frac{\partial^{3} \pi^{\perp} g_{1}}{\partial b^{2}}\left(z_{\alpha}\right) \gamma_{1}(\alpha)^{2} \odot \gamma_{2}(\alpha)+5 \frac{\partial^{4} \pi^{\perp} g_{1}}{\partial b^{4}}\left(z_{\alpha}\right) \gamma_{1}(\alpha)^{4} \\
& +20 \frac{\partial \pi^{\perp} g_{2}}{\partial b}\left(z_{\alpha}\right) \gamma_{3}(\alpha)+60 \frac{\partial^{2} \pi^{\perp} g_{2}}{\partial b^{2}}\left(z_{\alpha}\right) \gamma_{1}(\alpha) \odot \gamma_{2}(\alpha) \\
& +20 \frac{\partial^{3} \pi^{\perp} g_{2}}{\partial b^{3}}\left(z_{\alpha}\right) \gamma_{1}(\alpha)^{3}+60 \frac{\partial \pi^{\perp} g_{3}}{\partial b}\left(z_{\alpha}\right) \gamma_{2}(\alpha) \\
& \left.+60 \frac{\partial^{2} \pi^{\perp} g_{3}}{\partial b^{2}}\left(z_{\alpha}\right) \gamma_{1}(\alpha)^{2}+120 \frac{\partial \pi^{\perp} g_{4}}{\partial b}\left(z_{\alpha}\right) \gamma_{1}(\alpha)\right)
\end{aligned}
$$

The averaged functions, as stated in Theorem B, are computed as follows:

$$
g_{i}(z)=Y(T, z)^{-1} \frac{y_{i}(T, z)}{i !} .
$$

So, from the recurrence (12), we explicitly develop the expressions of $y_{i}$, for $i=0,1, \ldots, 5$.

$$
\begin{aligned}
& y_{0}(t, z)=x(t, z, 0)-z \\
& y_{1}(t, z)=Y(t, z) \int_{0}^{t} Y(\tau, z)^{-1} F_{1}(\tau, x(\tau, z, 0)) \mathrm{d} \tau \\
& y_{2}(t, z)=Y(t, z) \int_{0}^{t} Y(\tau, z)^{-1}\left[2 F_{2}(\tau, x(\tau, z, 0))+2 \frac{\partial F_{1}}{\partial x}(\tau, x(\tau, x, 0)) y_{1}(\tau, z)\right.
\end{aligned}
$$




$$
\begin{aligned}
& \left.+\frac{\partial^{2} F_{0}}{\partial x^{2}}(\tau, x(\tau, z, 0)) y_{1}(\tau, z)^{2}\right] \mathrm{d} \tau \\
& y_{3}(t, z)=Y(t, z) \int_{0}^{t} Y(\tau, z)^{-1}\left[6 F_{3}(\tau, x(\tau, z, 0))+6 \frac{\partial F_{2}}{\partial x}(\tau, x(\tau, x, 0)) y_{1}(\tau, z)\right. \\
& +3 \frac{\partial^{2} F_{1}}{\partial x^{2}}(\tau, x(\tau, z, 0)) y_{1}(\tau, z)^{2}+3 \frac{\partial F_{1}}{\partial x}(\tau, x(\tau, z, 0)) y_{2}(\tau, z) \\
& \left.+3 \frac{\partial^{2} F_{0}}{\partial x^{2}}(\tau, x(\tau, z, 0)) y_{1}(\tau, z) \odot y_{2}(\tau, z)+\frac{\partial^{3} F_{0}}{\partial x^{3}}(\tau, x(\tau, z, 0)) y_{1}(\tau, z)^{3}\right] \mathrm{d} \tau, \\
& y_{4}(t, z)=Y(t, z) \int_{0}^{t} Y(\tau, z)^{-1}\left[24 F_{4}(\tau, x(\tau, z, 0))+24 \frac{\partial F_{3}}{\partial x}(\tau, x(\tau, x, 0)) y_{1}(\tau, z)\right. \\
& +12 \frac{\partial^{2} F_{2}}{\partial x^{2}}(\tau, x(\tau, z, 0)) y_{1}(\tau, z)^{2}+12 \frac{\partial F_{2}}{\partial x}(\tau, x(\tau, z, 0)) y_{2}(\tau, z) \\
& +12 \frac{\partial^{2} F_{1}}{\partial x^{2}}(\tau, x(\tau, z, 0)) y_{1}(\tau, z) \odot y_{2}(\tau, z)+4 \frac{\partial^{3} F_{1}}{\partial x^{3}}(\tau, x(\tau, z, 0)) y_{1}(\tau, z)^{3} \\
& +4 \frac{\partial F_{1}}{\partial x}(\tau, x(\tau, z, 0)) y_{3}(\tau, z)+3 \frac{\partial^{2} F_{0}}{\partial x^{2}}(\tau, x(\tau, z, 0)) y_{2}(\tau, z)^{2} \\
& +4 \frac{\partial^{2} F_{0}}{\partial x^{2}}(\tau, x(\tau, z, 0)) y_{1}(\tau, z) \odot y_{3}(\tau, z) \\
& \left.+6 \frac{\partial^{3} F_{0}}{\partial x^{3}}(\tau, x(\tau, z, 0)) y_{1}(\tau, z)^{2} \odot y_{2}(\tau, z)+\frac{\partial^{4} F_{0}}{\partial x^{4}}(\tau, x(\tau, z, 0)) y_{1}(\tau, z)^{4}\right] \mathrm{d} \tau \\
& y_{5}(t, z)=Y(t, z) \int_{0}^{t} Y(\tau, z)^{-1}\left[120 F_{5}(\tau, x(\tau, z, 0))+120 \frac{\partial F_{4}}{\partial x}(\tau, x(\tau, x, 0)) y_{1}(\tau, z)\right. \\
& +60 \frac{\partial^{2} F_{3}}{\partial x^{2}}(\tau, x(\tau, z, 0)) y_{1}(\tau, z)^{2}+60 \frac{\partial F_{3}}{\partial x}(\tau, x(\tau, z, 0)) y_{2}(\tau, z) \\
& +60 \frac{\partial^{2} F_{2}}{\partial x^{2}}(\tau, x(\tau, z, 0)) y_{1}(\tau, z) \odot y_{2}(\tau, z)+20 \frac{\partial^{3} F_{2}}{\partial x^{3}}(\tau, x(\tau, z, 0)) y_{1}(\tau, z)^{3} \\
& +20 \frac{\partial F_{2}}{\partial x}(\tau, x(\tau, z, 0)) y_{3}(\tau, z)+20 \frac{\partial^{2} F_{1}}{\partial x^{2}}(\tau, x(\tau, z, 0)) y_{1}(\tau, z) \odot y_{3}(\tau, z) \\
& +15 \frac{\partial^{2} F_{1}}{\partial x^{2}}(\tau, x(\tau, z, 0)) y_{2}(\tau, z)^{2}+30 \frac{\partial^{3} F_{1}}{\partial x^{3}}(\tau, x(\tau, z, 0)) y_{1}(\tau, z)^{2} \odot y_{2}(\tau, z) \\
& +5 \frac{\partial^{4} F_{1}}{\partial x^{4}}(\tau, x(\tau, z, 0)) y_{1}(\tau, z)^{4}+5 \frac{\partial F_{1}}{\partial x}(\tau, x(\tau, z, 0)) y_{4}(\tau, z) \\
& +10 \frac{\partial^{2} F_{0}}{\partial x^{2}}(\tau, x(\tau, z, 0)) y_{1}(\tau, z) \odot y_{3}(\tau, z)
\end{aligned}
$$




$$
\begin{aligned}
& +5 \frac{\partial^{2} F_{0}}{\partial x^{2}}(\tau, x(\tau, z, 0)) y_{1}(\tau, z) \odot y_{4}(\tau, z) \\
& +15 \frac{\partial^{3} F_{0}}{\partial x^{3}}(\tau, x(\tau, z, 0)) y_{1}(\tau, z) \odot y_{2}(\tau, z)^{2} \\
& +10 \frac{\partial^{3} F_{0}}{\partial x^{3}}(\tau, x(\tau, z, 0)) y_{1}(\tau, z)^{2} \odot y_{3}(\tau, z) \\
& \left.+10 \frac{\partial^{4} F_{0}}{\partial x^{4}}(\tau, x(\tau, z, 0)) y_{1}(\tau, z)^{3} \odot y_{2}(\tau, z)+\frac{\partial^{5} F_{0}}{\partial x^{5}}(\tau, x(\tau, z, 0)) y_{1}(\tau, z)^{5}\right] \mathrm{d} \tau
\end{aligned}
$$

Appendix: BASIC RESUlts on the Brouwer DEGREe

In this appendix we present the existence and uniqueness result from the degree theory in finite dimensional spaces. We follow the Browder's paper [3], where are formalized the properties of the classical Brouwer degree. We also present some results that we shall need for proving the main results of this paper.

Theorem 8. Let $X=\mathbb{R}^{n}=Y$ for a given positive integer $n$. For bounded open subsets $V$ of $X$, consider continuous mappings $f: \mathrm{Cl}(V) \rightarrow Y$, and points $y_{0}$ in $Y$ such that $y_{0}$ does not lie in $f(\partial V)$ (as usual $\partial V$ denotes the boundary of $V$ ). Then to each such triple $\left(f, V, y_{0}\right)$, there corresponds an integer $d\left(f, V, y_{0}\right)$ having the following three properties.

(i) If $d\left(f, V, y_{0}\right) \neq 0$, then $y_{0} \in f(V)$. If $f_{0}$ is the identity map of $X$ onto $Y$, then for every bounded open set $V$ and $y_{0} \in V$, we have

$$
d\left(\left.f_{0}\right|_{V}, V, y_{0}\right)= \pm 1
$$

(ii) (Additivity) If $f: \mathrm{Cl}(V) \rightarrow Y$ is a continuous map with $V$ a bounded open set in $X$, and $V_{1}$ and $V_{2}$ are a pair of disjoint open subsets of $V$ such that

$$
y_{0} \notin f\left(\mathrm{Cl}(V) \backslash\left(V_{1} \cup V_{2}\right)\right)
$$

then,

$$
d\left(f_{0}, V, y_{0}\right)=d\left(f_{0}, V_{1}, y_{0}\right)+d\left(f_{0}, V_{1}, y_{0}\right) .
$$

(iii) (Invariance under homotopy) Let $V$ be a bounded open set in $X$, and consider a continuous homotopy $\left\{f_{t}: 0 \leq t \leq 1\right\}$ of maps of $\mathrm{Cl}(V)$ in to $Y$. Let $\left\{y_{t}: 0 \leq t \leq 1\right\}$ be a continuous curve in $Y$ such that $y_{t} \notin f_{t}(\partial V)$ for any $t \in[0,1]$. Then $d\left(f_{t}, V, y_{t}\right)$ is constant in $t$ on $[0,1]$.

Theorem 9. The degree function $d\left(f, V, y_{0}\right)$ is uniquely determined by the three conditions of Theorem 8.

For the proofs of Theorems 8 and 9 see [3]. 


\section{ACKNOWLEDGEMENTS}

The first author is partially supported by CNPq 248501/2013-5. The second author is partially supported by the MINECO grants MTM2016-77278-P and MTM2013-40998-P, an AGAUR grant number 2014SGR-568, the grants FP7PEOPLE-2012-IRSES 318999, and a CAPES-MECD grant PHB-2009-0025-PC. The third author is supported by FAPESP grants 2015/02517-6, 2015/24841-0, and 2016/11471-2. The second and the third authors are supported by the European Community FP7-PEOPLE-2012-IRSES-316338 and FP7-PEOPLE-2012IRSES-318999 grants and by the CAPES grant number 88881.030454/2013-01 from the program CSF-PVE.

\section{REFERENCES}

[1] H. Amann and G. Metzen, Ordinary Differential Equations: An Introduction to Nonlinear Analysis, Degruyter Studies in Mathematics 13, De Degruyter, Berlin 1990.

[2] F. T. ARECCHI, Chaos and generalized multistability in quantum optics, Physica Scripta 9 (1985), 85-92.

[3] F. Browder, Fixed point theory and nonlinear problems, Bull. Amer. Math. Soc. 9 (1983), $1-39$.

[4] A. Buicŭ, J.P. Françoise And J. Llibre, Periodic solutions of nonlinear periodic differential systems with a small parameter, Communications on Pure and Applied Analysis 6 (2007), 103-111.

[5] A. Buică,J. Giné And J. Llibre, A second order analysis of the periodic solutions for nonlinear periodic differential systems with a small parameter, Physica D: Nonlinear Phenomena 241 (2012), 528-533.

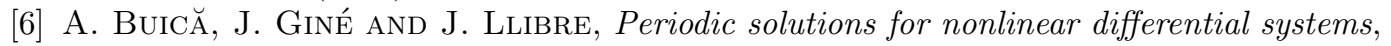
Topological Methods in Nonlinear Analysis 43 (2014), 403-419.

[7] M.R. CÂNDIDO AND J. Llibre, New results on averaging theory and applications, Z. Angew. Math. Phys. 67 No. 4, 1-11, (2016).

[8] C. Chicone, Lyapunov-Schmidt reduction and Melnikov integrals for bifurcation of perioric solutions in coupled oscillators, J. Differential Equations 112 (1994), 407-447.

[9] J. Giné, M. Grau And J. LliBRe, Averaging theory at any order for computing periodic orbits, Physica D 250 (2013), 58-65.

[10] J. Giné, J. Llibre, K. Wu, And X. Zhang, Averaging methods of arbitrary order, periodic solutions and integrability, J. Differential Equations 260 (2016), 4130-4156.

[11] J. Llibre, D. D. Novaes And M. A. Teixeira, Higher order averaging theory for finding periodic solutions via Brouwer degree, Nonlinearity, 27 (2014), 563-583.

[12] W. P. Johnson, The curious history of Faá di Bruno's formula, The American Mathematical Monthly 109, No. 3 (2002), 217-234.

[13] L. Liu, O. O. Aybar, V. G. Romanovski And W. Zhang, Identifying weak foci and centers in Maxwell-Bloch system, J. Math. Anal. Appl. 430 (2015), 549-571.

[14] J. LliBRE AND D.D. NovAES, Improving the averaging theory for computing periodic solutions of the differential equations, Zeitschrift für angewandte Mathematik und Physik 66 (2015), 1401-1412.

[15] D.D. Novaes, An equivalent formulation of the averaged functions via Bell polynomials, Trends in Mathematics: Research Perspectives CRM Barcelona (Springer), accepted August 2016. 
[16] J. A. Sanders F. Verhulst And J. Murdock, Averaging Methods in Nonlinear Dynamical Systems, Second edition, Applied Mathematical Sciences 59, Springer, New York, 2007.

[17] I.G. Malkin, Some problems of the theory of nonlinear oscillations, (Russian) Gosudarstv. Izdat. Tehn.-Teor. Lit., Moscow, 1956.

[18] M. Roseau, Vibrations non linéaires et théorie de la stabilité, (French) Springer Tracts in Natural Philosophy, Vol.8 Springer-Verlag, Berlin-New York, 1966.

${ }^{1}$ Departamento de Matemática, Universidade Estadual de Campinas, Rua Sérgio Baruque de Holanda, 651, Cidade Universitária Zeferino Vaz, 13083-859, CampINAS, SP, BRAZIL

E-mail address: ddnovaes@ime.unicamp.br

2 Departament de Matemàtiques, Universitat Autònoma de Barcelona, 08193 Bellaterra, Barcelona, Catalonia, Spain

E-mail address: candidomr@mat.uab.cat, jllibre@mat.uab.cat 